\title{
Numerical solution of axisymmetric, unsteady free-boundary problems at finite Reynolds number. II. Deformation of a bubble in a biaxial straining flow
}

\author{
I. S. Kang and L. G. Leal \\ Department of Chemical Engineering, California Institute of Technology, Pasadena, California 91125
}

(Received 11 August 1988; accepted 28 November 1988)

\begin{abstract}
Numerical solutions of the full Navier-Stokes equations are used to investigate the steady and unsteady deformation of a bubble in a biaxial straining flow for Reynolds numbers in the range $0 \leqslant R \leqslant 400$, and Weber numbers up to $O(10)$. The steady-state bubble shape and the frequency of small amplitude oscillations of shape are both identical for biaxial and uniaxial straining flows in the potential flow limit. However, for a large, but finite Reynolds number, the bubble shape in the biaxial straining flow is found to be fundamentally different from the shape in uniaxial flows. This is shown to be a consequence of vorticity enhancement via vortex line stretching in the biaxial flow, which does not occur in the uniaxial flow. At the highest Reynolds number considered here, $R=400$, the steady-state bubble behavior for low $W$ is qualitatively similar to the potential flow case, with a limit point for existence of the low $W$ branch of steady solutions occurring at $W \sim 6$. However, in this case a second branch of steady solutions is found for larger $W>7$, which exhibits oblate bubble shapes for large $W$, and has no counterpart in the potential flow limit. In unsteady flows, the behavior of bubble deformation is fundamentally different in the uniaxial and biaxial flows for both high Reynolds numbers and the potential flow limit. This suggests that breakup will occur in far different ways in the two cases.
\end{abstract}

\section{INTRODUCTION}

An outstanding, largely unresolved problem of fluid mechanics is the conditions for breakup of bubbles or drops in flows at large or moderate Reynolds numbers. This is not because the problem is unimportant. Indeed, theoretical models for bubble size distributions in blenders, mixers, and a variety of more general two-phase flows requires an a priori prediction of a breakup criteria. The main problem from an experimental point of view is the difficulty of observation in well-characterized flows, while theoretical analyses have been restricted by the lack of an effective solution method for large deformation free-boundary problems at finite Reynolds numbers. Generally, in the mainly empirical results that have been reported to date (cf. Hinze ${ }^{1}$ or Lewis and Davidson $^{2}$ ), it has been assumed that breakup will occur at a critical Weber number that is independent of the details of the flow.

Here, we take advantage of the recent development of an effective numerical method for solution of free-boundary problems at a finite Reynolds number to pursue a fundamental, theoretical investigation of bubble and drop breakup at moderate and large Reynolds numbers. Specifically, the present paper is the second in a series (see Kang and Leal, ${ }^{3}$ hereafter denoted by $I$ ) in which we use numerical methods to study bubble deformation in axisymmetric flow fields at finite Reynolds numbers $R$. In I, we considered uniaxial straining flow. Here we consider the same problem for a biaxial straining flow. From a mathematical point of view, the two problems are identical except for a change in sign in the velocity at infinity, and the predicted steady-state bubble shapes for the two types of flow are identical in the potential flow limit. However, we shall see that the simple change in the far-field boundary condition can result in fundamentally different bubble behavior for finite $R$. Specifically, whereas a limit point is observed for the existence of steady axisymmetric bubble shapes at a Weber number of $O(2-3)$ in uniaxial flow for all $R \geqslant 10$, a corresponding limit point was not found for biaxial flow at any $W \leqslant O(10)$ for $R$ up to 200 . This strongly suggests that the flow type plays a critical role in bubble breakup, in contrast to the assumptions of previous studies.

The deformation of a bubble in a uniaxial straining flow has been studied extensively via numerical analyses ${ }^{3-6}$ as well as by approximate analytic theories, ${ }^{7-14}$ and is now fairly well understood. The most important result is that steady bubble shapes exist only for Weber numbers below a certain critical value $W_{c}$, which depends on the Reynolds number. This was first suggested by Miksis ${ }^{5}$ and Ryskin and Leal, ${ }^{6}$ based on the breakdown of steady numerical solution algorithms, and was later supported in I by full transient solutions which showed that the breakdown of the steady solutions occurred precisely at the point of onset of a "waist" in the bubble shape, and was followed by a continuous stretching of the bubble along the principal axis of strain. In I, and in a companion paper, ${ }^{13}$ we have also examined oscillations in the bubble shape for a steady uniaxial, straining flow. The basic conclusion from these works is that the frequency of the lowest mode $\left[P_{2}(\cos \theta)\right]$ decreases monotonically as the Weber number increases, reaching zero at a critical Weber number that is identical to the limit point of the steady solution branch. Besides being of interest in its own right, this provides further evidence for the existence of the limit point for steady solutions. Since the governing equations for small oscillations are identical to the linearized equations for stability of the steady state, the existence of a zero frequency point (zero eigenvalue) implies a singularity in the Jacobian matrix for evolution of a disturbance about the steady state. 
In contrast to the uniaxial straining flow problem, relatively little is known about the behavior of a deforming bubble in a biaxial straining flow. Indeed, we are aware of only one analysis for this problem by Frankel and Acrivos ${ }^{14}$ who calculated the bubble shape in the creeping flow limit to a moderate value of capillary number $(\mathrm{Ca} \leqslant 0.2)$. Although Hinze, ${ }^{1}$ and Lewis and Davidson ${ }^{2}$ also mention the biaxial flow problem at higher Reynolds numbers, they do not provide any analysis. We can only surmise that previous investigators have not examined biaxial flows because they assumed that the results would not differ in any fundamental way from the uniaxial flow problem. In this paper, we consider both steady and unsteady deformations of a bubble in a biaxial straining flow at finite Reynolds numbers. As we shall see, there are extremely important fundamental differences between the uniaxial and biaxial flow problems.

\section{STATEMENT OF THE PROBLEM}

We consider the steady and unsteady deformation of an incompressible gas bubble of volume $\frac{4}{3} \pi a^{3}$ in a biaxial straining flow of a fluid with constant density $\rho$ and constant viscosity $\mu$ (see Fig. 1). The density and viscosity of the gas inside the bubble are assumed to be negligible in comparison with those of the liquid. Furthermore, the surface of the bubble is assumed to be characterized completely by a uniform surface tension $\gamma$. Finally, we neglect all effects of gravity including the hydrostatic pressure variation in the fluid. If the $x$ axis of a cylindrical coordinate system $(x, \sigma, \phi)$ is directed along the axis of symmetry, the (dimensional) velocity field far from the bubble is given by

$$
\mathbf{u}=\mathbf{E} \cdot \mathbf{r}, \quad \mathbf{E}=E\left(\begin{array}{ccc}
-1 & 0 & 0 \\
0 & \frac{1}{2} & 0 \\
0 & 0 & \frac{1}{2}
\end{array}\right), \quad E>0,
$$

where $E$ is the principal strain rate. We use the equivalent radius $a$ of the bubble as a characteristic length scale, the product $E a$ as a characteristic velocity scale, and $E^{-1}$ as a characteristic time scale. The relevant dimensionless parameters in this problem are the Reynolds number $R \equiv 2 \rho(E a) a / \mu$, based on the equivalent diameter $2 a$ of the bubble, and the Weber number $W \equiv 2 \rho(E a)^{2} a / \gamma$.

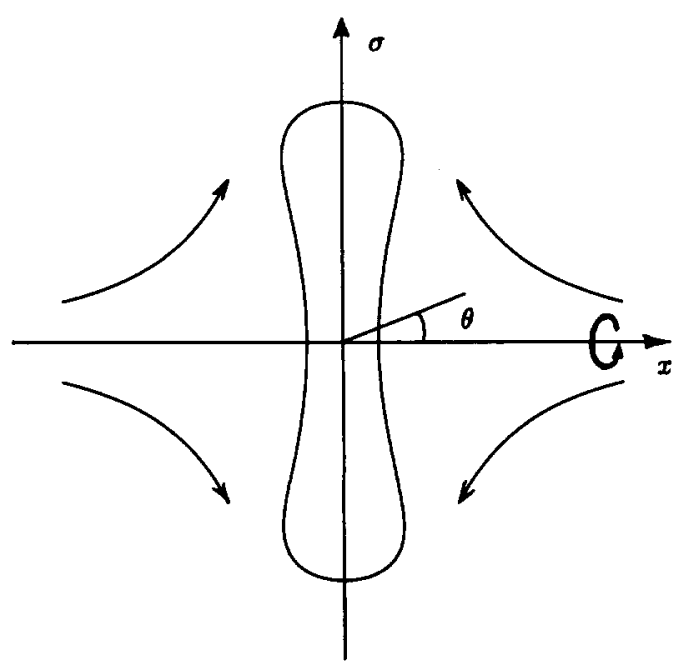

FIG. 1. A bubble in a biaxial straining flow.
From a mathematical point of view, the biaxial straining flow problem is exactly the same as the uniaxial straining flow problem, except that the signs are changed in the boundary condition at infinity [Eq. (1)]. However, as we shall see, this simple change in boundary conditions results in fundamentally different solution behavior.

\section{PRELIMINARY ANALYTICAL RESULTS}

In this section, we present several analytical results that can be obtained without any detailed numerical analysis. These results will play an important role in understanding and interpreting the numerical computations that will be considered in later sections.

\section{A. Steady-state shape and oscillation frequency of a bubble in potential flow}

We begin by considering bubble deformation in the potential flow limit. In this limit, we first assert that the steadystate shape of a bubble is identical in biaxial and uniaxial straining flows. In itself, this assertion may not seem particularly noteworthy, and we shall see that its proof is trivial. However, it is well known ${ }^{5,6,13}$ that a bubble in a uniaxial straining flow is elongated in the axial direction [i.e., $f(0)>f(\pi / 2)$ when the surface is given by $r=1+f(\theta)]$, and the identity of shape between uniaxial and biaxial flows implies that the bubble should be elongated in the same direction in a biaxial flow. This conclusion does seem quite surprising at first, because it implies the counterintuitive result of bubble elongation against the primary flow direction.

To prove that the steady-state shape must be identical in uniaxial and biaxial potential flow, we need only to examine the dimensionless governing equations and boundary conditions for the steady-state potential flow. The governing equation is

$$
\nabla^{2} \phi=0 .
$$

For the boundary conditions at the bubble surface $[r=1+f(\theta)]$, we have the kinematic condition

$$
\boldsymbol{\nabla} \phi \cdot \mathbf{n}=0,
$$

and the normal stress condition

$$
G+\nabla \phi \cdot \nabla \phi=(4 / W) \nabla \cdot \mathbf{n} .
$$

In (4), the unknown constant $G$ must be determined from the condition of volume conservation. The far-field condition corresponding to the velocity field (1) is given by

$$
\phi \rightarrow \pm \frac{1}{4}\left(3 \cos ^{2} \theta-1\right) r^{2}, \quad \text { as } r \rightarrow \infty,
$$

where " + " and " - " refer to the uniaxial and the biaxial straining flows, respectively [hereafter the upper and lower signs of \pm (or $\mp$ ) refer to the uniaxial and biaxial straining flows, respectively].

Now, it is a trivial matter to show that for any given $W$ where a steady shape exists, exactly the same steady-state shapes must be obtained for both flow types. Let us assume that a steady-state shape exists (denoted as $f_{1}$ ) for the uniaxial flow problem, with a corresponding velocity potential $\left(\phi_{1}\right)$, so that the pair $\left(f_{1}, \phi_{1}\right)$ represents a solution of $(2)-$ (5) for the given $W$ [in fact, the velocity potential can be determined uniquely for a given shape from (2), (3), and (5), but the uniqueness is not necessary in this proof]. Now, our assertion is that $\left(f_{1},-\phi_{1}\right)$ is a solution for the biaxial 
straining flow problem. Clearly, $\phi=-\phi_{1}$ satisfies the farfield condition ( 5 ), the governing equation ( 2 ), and boundary condition (3), i.e.,

$$
\begin{aligned}
& \nabla^{2}\left(-\phi_{1}\right)=-\nabla^{2} \phi_{1}=0, \\
& \nabla\left(-\phi_{1}\right) \cdot \mathbf{n}=-\nabla \phi_{1} \cdot \mathbf{n}=0 .
\end{aligned}
$$

The left-hand side of the normal stress condition, on the other hand, does not change sign upon transformation from $\phi_{1}$ to $-\phi_{1}$

$$
\begin{aligned}
G+ & \nabla\left(-\phi_{1}\right) \cdot \nabla\left(-\phi_{1}\right) \\
& =G+\nabla \phi_{1} \cdot \nabla \phi_{1}=\left.(4 / W) \nabla \cdot \mathbf{n}\right|_{r=1+f_{1}} .
\end{aligned}
$$

Thus if $f_{1}$ is a solution for the shape for $\phi=\phi_{1}$, it is also a solution of (8) for $\phi=-\phi_{1}$. This proves our assertion. In physical terms, the change in direction of the steady-state potential flow does not result in any shape change, because the dynamic pressure distribution $\left(-u^{2}+\right.$ const along a streamline) remains unchanged.

In spite of the fact that steady-state shapes for uniaxial and biaxial flow are identical, a relationship between transient shapes in uniaxial and biaxial flow could only be obtained for $W \ll 1$. In particular, for this limit the oscillation frequency of the primary deformation mode $\left[P_{2}(\cos \theta)\right]$ is identical in the two types of flow. Kang and Leal ${ }^{13}$ showed that the oscillation frequency of the primary deformation mode of a bubble in a uniaxial straining flow decreases as the Weber number increases, i.e.,

$$
\omega_{2}^{2}=\omega_{2,0}^{2}(1-0.31 W)+o(W),
$$

where $\omega_{2,0}$ is the frequency of the primary mode for an oscillating bubble in an otherwise quiescent fluid. Here we present only the minimum analysis necessary to show that (9) is also true for the biaxial straining flow problem. For this purpose, we only need to introduce characteristic velocity, length, and time scales that are appropriate to the oscillation problem, ${ }^{13}$

$$
\tilde{\phi}_{c}=(\gamma a / \rho)^{1 / 2}, \quad \tilde{t}_{c}=\left(\rho a^{3} / \gamma\right)^{1 / 2}, \quad l_{c}=a .
$$

The result ${ }^{13}$ is that the oscillation problem for either uniaxial or biaxial flow is characterized by a single dimensionless parameter, $\pm \sqrt{W}$, which appears in the far-field boundary condition for the two types of flow,

$$
\tilde{\phi}_{\infty}= \pm \sqrt{W / 2}\left(3 \cos ^{2} \theta-1\right)\left(\frac{1}{4} r^{2}\right),
$$

where " + " and " - " of " \pm " refer to the uniaxial and the biaxial straining flows, respectively. However, the oscillation frequency (9), for the uniaxial case, depends on the square of this parameter. Hence if we designate a dimensionless parameter $\xi$ as being $+\sqrt{W}$ for uniaxial flow, and $-\sqrt{W}$ for biaxial flow, it is evident that the result (9) must have the invariant form

$$
\omega_{2}^{2}=\omega_{2,0}^{2}\left(1-0.31 \xi^{2}\right)+o\left(\xi^{2}\right),
$$

for both types of flow.

\section{B. Steady bubble shape for $W \ll 1$ and large, but finite Reynolds number}

A bubble in a biaxial straining flow, as shown in Sec. III A, has a steady-state shape in the potential flow approxi- mation that is elongated in the axial direction. On the other hand, a bubble in the creeping flow limit is known to deform into an oblate shape in a biaxial straining flow. Thus, provided that the potential flow solution is the solution for the real flow in the limit $R \rightarrow \infty$, there must be an intermediate Reynolds number where the transition from oblate to prolate shapes takes place. We call this the marginal Reynolds number $R_{m}$. In this section, we obtain an analytic estimate for $R_{m}$ in the limit of a small deformation, $W \ll 1$, by solving for the bubble shape at large, but finite $R$.

Let us begin by writing the normal stress condition in a form that is accurate to $O\left(R^{-1}\right)$,

$$
\begin{aligned}
-2 p_{v}+\nabla \phi_{p} \cdot \nabla \phi_{p}+\frac{8}{R} & \frac{\partial^{2} \phi_{p}}{\partial n^{2}} \\
= & \frac{4}{W} \nabla \cdot \mathbf{n}, \quad \text { at } \quad r=1+f(\theta),
\end{aligned}
$$

where $p_{v}$ denotes the dimensionless viscous pressure correction defined as $p_{v}=p_{v}^{\prime} /\left(\rho U_{c}^{2}\right)$ and $\phi_{p}$ denotes the steadystate velocity potential. If we restrict ourselves to the limit of small deformation with $W \ll 1$, a first approximation to the shape can be calculated by solving (11) with the left-handside evaluated using the solution for a spherical bubble.

To do this, we must first evaluate the viscous pressure correction. For this purpose, we can use the general formula for the viscous pressure correction around a spherical bubble that was derived in our earlier paper ${ }^{13}$ under the assumption that the velocity perturbation resulting from viscosity approaches zero everywhere as $R \rightarrow \infty$, i.e.,

$$
\left\|\mathbf{u}_{v}\right\| \ll\left\|\mathbf{u}_{p}\right\|, \text { for } R \gg 1 \text {. }
$$

Here, $\mathbf{u}_{p}$ and $\mathbf{u}_{v}$ denote the potential flow velocity and the velocity perturbation resulting from viscosity, respectively. Since the vorticity is solenoidal $(\nabla \cdot \omega=0)$, it can be expressed in terms of toroidal $(T)$ fields for any arbitrary axisymmetric problem,

$$
\omega=\mathbf{T}=\nabla \times\left(\sum_{n=0}^{\infty} T_{n}(r, t) P_{n}(\cos \theta) \mathbf{e}_{r}\right) .
$$

Then the leading-order viscous pressure correction at the surface of a spherical bubble in an arbitrary axisymmetric flow (steady or unsteady) at a high Reynolds number is given by (see Kang and Leal ${ }^{13}$ for a detailed derivation)

$$
\begin{aligned}
p_{v}(1, \theta, t)= & \sum_{n=0}^{\infty}\left[n \int_{1}^{\infty} r^{-n-1} \sum_{k=-\infty}^{\infty} f_{n, k}(r) T_{n+k} d r\right. \\
& +\int_{1}^{\infty} \sum_{l=-\infty}^{\infty}\left\{\left[r^{-n} g_{n, l}(r)\right.\right. \\
& \left.\left.-r^{-n-t} g_{n, l}(1)\right] T_{n+l}\right\} d r \\
& \left.+n\left(\frac{2}{R}\right) T_{n}(1, t)\right] P_{n}(\cos \theta),
\end{aligned}
$$

where

$$
\begin{aligned}
\sum_{n=0}^{\infty}( & \left.\sum_{k=-\infty}^{\infty} f_{n, k} T_{n+k}\right) P_{n} \\
\quad= & \sum_{n=0}^{\infty}\left[\left(u_{p r} P_{n}-\int_{0}^{\theta} P_{n} \frac{\partial u_{p r}}{\partial \theta} d \theta\right) T_{n}\right],
\end{aligned}
$$


$\sum_{n=0}^{\infty}\left(\sum_{l=-\infty}^{\infty} g_{n, l} T_{n+1}\right) P_{n}=\sum_{n=0}^{\infty}\left(\frac{T_{n}}{r} u_{p \theta} \frac{d P_{n}}{d \theta}\right)$

Now, we want to estimate the first-order deformation with respect to $W$ (for $W \ll 1$ ) using (11), with the left-handside evaluated using (13) and the steady-state potential flow solution for the spherical bubble in uniaxial and biaxial straining flows,

$$
\phi_{p}= \pm P_{2}(\cos \theta)\left(\frac{1}{2} r^{2}+\frac{1}{3} r^{-3}\right) .
$$

As we can see in (13), however, an exact evaluation of the pressure correction on the surface of the bubble requires the vorticity distribution in the region outside the bubble, and this would require us to solve the vorticity equation. Instead, for present purposes we adopt the ad hoc approximation that the vorticity is strictly confined to the interface and is zero everywhere else in the fluid. [ In fact, the contribution of the vorticity distributed inside the boundary layer is of the same magnitude, $O\left(R^{-1}\right)$, as that of the vorticity at the interface, i.e., as the last term in (13). The neglect of the boundarylayer contribution to (13) will be justified by comparing the estimated shapes with the full numerical solution in Fig. 2.] With this ad hoc approximation, (13) reduces to

$$
p_{v}(1) \simeq \sum_{n=0}^{\infty} n\left(\frac{2}{R}\right) T_{n}(1) P_{n}(\cos \theta) .
$$

The coefficients $T_{n}(1)$ are determined, via Eq. (12), from the vorticity distribution at the bubble surface. The surface vorticity distribution is obtained using the condition of vanishing shear stress, i.e.,

$$
\omega(1, \theta)=\left.2\left(\frac{u_{p \theta}}{r}-\frac{1}{r} \frac{\partial u_{p r}}{\partial \theta}\right)\right|_{r=1}+o(1),
$$

with the velocity components calculated from the potential flow approximation. The result for $\omega(1, \theta)$ is

$$
\omega(1, \theta)= \pm \frac{5}{3} \frac{d P_{n}}{d \theta}
$$

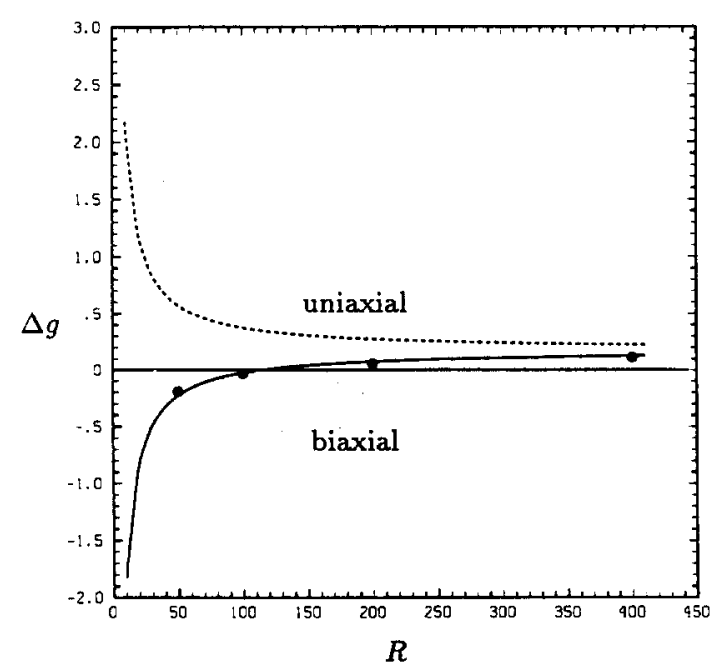

FIG. 2. Here, $\Delta g=g(0)-g(\pi / 2)$ in terms of the Reynolds number, where the shape of the bubble is given by $r_{s}(\theta)=1+(W / 4) g(\theta)(一$ : small deformation analysis for biaxial straining flow; ...: small deformation analysis for uniaxial straining flow; 9 : full numerical solution for biaxial straining flow).
Comparing this with Eq. (12), we find

$$
T_{2}(1)=\mp 5 / 3
$$

and

$$
T_{n}(1)=0, \text { for } n \neq 2 \text {. }
$$

Finally, by substituting (14)-(16) into the left-hand-side of (11), we obtain

$$
\begin{aligned}
\boldsymbol{\nabla} \cdot \mathbf{n}= & 2+\frac{W}{4}\left[-\frac{10}{7} P_{4}(\cos \theta)\right. \\
& \left.+\left(\frac{25}{42} \pm \frac{160}{3 R}\right) P_{2}(\cos \theta)\right] .
\end{aligned}
$$

To solve this equation for a first-order approximation to the bubble shape (for $W \ll 1$ ), let us assume that the bubble surface is described in the form

$r=1+f=1+\frac{W}{4} g(\theta)=1+\frac{W}{4} \sum_{n=2}^{\infty} \beta_{n} P_{n}(\cos \theta)$.

Then, the curvature is given by

$$
\begin{aligned}
\boldsymbol{\nabla} \cdot \mathrm{n}=2 & +\frac{W}{4} \sum_{n=2}^{\infty}(n-1)(n+2) \beta_{n} P_{n}(\cos \theta) \\
& +O\left(W^{2}\right) .
\end{aligned}
$$

Finally, by comparing (17) and (19), we obtain

$g(\theta)=\left(\frac{25}{168} \pm \frac{40}{3 R}\right) P_{2}(\cos \theta)-\frac{5}{63} P_{4}(\cos \theta)$.

The solution (20) for the bubble shape is identical in the limit $R \rightarrow \infty$ with the small deformation solution obtained for potential flow in Ref. 13. As noted in Sec. III A, the potential flow limit of $(20)$ does not depend upon whether the undisturbed flow is uniaxial or biaxial in form. In both cases, the shape is prolate, with the $P_{4}$ term contributing a modification from the prolate ellipsoid $\left(P_{2}\right)$ toward a barrellike shape, with maximum radii occurring between the axis of symmetry and the equatorial plane. The term $\pm(40 /$ $3 R) P_{2}(\cos \theta)$ represents the leading-order viscous contribution to the bubble shape. Although the functional form is the same for the biaxial and uniaxial flows, the sign of this term is positive in the uniaxial case and negative in the biaxial case. Thus in the uniaxial flow, the effect of viscosity (i.e., finite $R$ ) is to make the bubble more prolate, while the contribution in biaxial flow is in the opposite direction.

In view of the fact that the bubble shape in biaxial flow at small $R$ is oblate, the tendency of the viscous shape correction toward an oblate shape may be viewed as "expected." Nevertheless, it is of interest to dig slightly below the surface to consider the mechanism of the viscous modification of shape. In fact, the viscous term in (20) has two sources, both of the same sign. First, the vorticity generated at the bubble surface is swept downstream (i.e., toward the axis of symmetry for the uniaxial flow, and toward the equatorial plane for the biaxial flow), and this causes the pressure to decrease in that region relative to its potential flow values $(10 / 3 R$ of 40 / $3 R$ is from this effect). Second, there is a viscous normal stress contribution, coming from the third term on the lefthand-side of Eq. (11), which has the same sign (30/3R of $40 / 3 R$ is from this effect). Thus the total normal force at the 
interface is decreased near the axis of symmetry for the uniaxial flow, and the bubble elongates slightly in that direction. For the biaxial flow, on the other hand, the total normal force at the interface decreases near the equatorial plane, and the bubble responds by adopting a less prolate shape.

One qualitative result from our earlier studies of bubble deformation and breakup at both high and low Reynolds numbers, is that "breakup" (more accurately continuous elongation of bubble shape) occurs when the bubble develops a "waist," and in all cases examined to date this corresponds to achieving a sufficiently prolate shape. Thus in uniaxial flow, where the viscous correction in (20) produces a more elongated shape with a decrease in $R$, one might surmise that a decrease in $R$ should cause the bubble to be more susceptible to breakup via the continuous stretching mechanism. In fact, this trend was found numerically by Ryskin and Leal ${ }^{6}$ who showed that the critical Weber number for breakup actually increased as $R$ increased. On the other hand, the small perturbation theory presented here suggests that a decrease in $R$ for a biaxial flow will cause a bubble to be less prolate, and thus less susceptible to breakup than for $R \rightarrow \infty$. In other words, we might expect for biaxial flow that the critical Weber number should actually decrease toward the potential flow limit as $R$ is increased. We shall see that this trend toward greater stability for finite $R$ is, in fact, observed in the numerical results obtained later. However, provided that the assumptions underlying the theory are correct (particularly the assumption $\left\|\mathbf{u}_{v}\right\| \ll\left\|\mathbf{u}_{p}\right\|$ ), another implication is that there should be a smooth transition with increase of $R$ to the potential flow limit for both uniaxial and biaxial flows. This conclusion is correct for small deformations of shape ( $W \ll 1$ ), but the numerical results show that it does not carry over in any simple way to cases involving finite deformation, at least up to $R=400$.

Finally, we may ask whether the approximation theory presented here can predict the transition Reynolds number $\boldsymbol{R}_{m}$ between oblate and prolate shapes, in accord with our initial goal. Roughly speaking, the transition point can be said to occur when

$$
\Delta g \equiv g(0)-g(\pi / 2)
$$

passes through zero [see the definition of $g(\theta)$ in (18)]. Now, according to $(20)$, the deformation along the symmetry axis $(\theta=0)$ is

$$
g(0)=5 / 72 \pm 40 / 3 R,
$$

and the deformation along the equatorial plane $(\theta=\pi / 2)$ is

$$
g(\pi / 2)=-5 / 48 \mp 20 / 3 R \text {. }
$$

Therefore

$$
\Delta g \equiv g(0)-g(\pi / 2)=25 / 144 \pm 20 / R,
$$

where " +" and "- " of \pm indicate the uniaxial and the biaxial straining flows, respectively. The $\Delta g$ 's for both types of flow are plotted as functions of $R$ in Fig. 2, where the $\Delta g$ 's obtained from full numerical solution are also presented for the biaxial flow case. The Weber numbers for the numerical solutions are chosen to correspond to small deformation cases ( $W=0.5$ for $R=50,100,200$, and 400 ), and the numerical results show good agreement with the asymptotic results. From Fig. 2, we can see that the bubble in a uniaxial straining flow always becomes less elongated as the Reynolds number increases, which is qualitatively consistent with the numerical solution of Ryskin and Leal. ${ }^{6}$ However, in a biaxial straining flow, the elongation direction is predicted to change from oblate to prolate at a marginal Reynolds number, $R_{m}=115.2$. As we can see by the comparison with numerical results in Fig. 2, this is a relatively good estimate of $R_{m}$ for small $W$.

\section{Vorticity stretching and the vorticity distribution in the wake}

The small deformation theory of Sec. III B demonstrates a qualitative difference between bubble deformation in uniaxial and biaxial extensional flows for large, but finite $R$. In particular, the advection of vorticity at the bubble surface produces a decrease in pressure in the "downstream" direction, which causes less prolate shapes for biaxial extension and more prolate shapes for uniaxial extension. Although the changes in shape are small in the perturbation theory, the fact that they are largely associated with vorticity produced at the bubble surface means that one may expect increased sensitivity to flow type for finite $W$, where the bubble will be more deformed and surface vorticity will be larger. Indeed, if we believe that the vorticity has a major role in determining bubble shape, as the small deformation theory suggests, then we may anticipate a rather profound difference between bubble deformation in uniaxial and biaxial flows. This is because there is active vorticity enhancement in the biaxial case, as a result of vortex line stretching, which has no counterpart in the uniaxial extensional flow.

To demonstrate that the assertions of the preceding paragraph are true, we may examine the vorticity transport equation for an axisymmetric inviscid flow without swirl, which takes the following simple form (Batchelor ${ }^{15}$ ):

$$
\frac{D(\omega / \sigma)}{D t}=0
$$

In fact, Eq. (21) is equivalent to Kelvin's circulation theorem. Equation (21) suggests that vorticity generated at the boundaries can grow indefinitely in an inviscid, biaxial straining flow, because a fluid particle travels in the direction of increasing $\sigma \rightarrow \infty$ as $t \rightarrow \infty$. For a uniaxial flow, on the other hand, the particle path is toward decreasing values of $\sigma$, so that the vorticity in an inviscid flow would become smaller. This suggests a fundamental and very important difference between uniaxial and biaxial flows, essentially resulting from the possibility of vortex stretching by the flow in one case, but not in the other. Now, the following question can be cast. Can the vorticity grow indefinitely in the downstream direction for a biaxial straining flow if the Reynolds number is large but finite? This question is answered by the following analysis for the wake region.

Let us begin by writing the vorticity transport equation in the cylindrical coordinate system that is illustrated in Fig. 1 , 


$$
\begin{aligned}
& u_{x} \frac{\partial \omega}{\partial x}+u_{\sigma} \frac{\partial \omega}{\partial \sigma}-\frac{\omega u_{\sigma}}{\sigma} \\
& =\frac{2}{R}\left[\frac{\partial^{2} \omega}{\partial x^{2}}+\frac{1}{\sigma} \frac{\partial}{\partial \sigma}\left(\sigma \frac{\partial \omega}{\partial \sigma}\right)-\frac{\omega}{\sigma^{2}}\right] .
\end{aligned}
$$

For $R \gg 1$, the vorticity is assumed to be confined to a thin layer with a thickness that we denote as $O(\delta)$ near the equatorial plane (i.e., wake). Then, in the wake region, $x=O(\delta), \sigma=O(1), u_{x}=O(\delta)$, and $u_{\sigma}=O(1)$. Now, let us introduce the rescaled variables such that

$$
\tilde{u}_{x}=u_{x} / \delta, \quad \tilde{x}=x / \delta .
$$

Then Eq. (22) becomes

$$
\tilde{u}_{x} \frac{\partial \omega}{\partial \tilde{x}}+u_{\sigma} \frac{\partial \omega}{\partial \sigma}-\frac{\omega u_{\sigma}}{\sigma}=\frac{2}{R}\left(\frac{1}{\delta^{2}} \frac{\partial^{2} \omega}{\partial \tilde{x}^{2}}+O(1)\right) .
$$

Therefore the most appropriate choice for $\delta$ is

$$
\delta=2 / \sqrt{R} .
$$

The introduction of a new variable $\Omega \equiv \omega / \sigma$ further reduces the equation to the following simple form:

$$
\tilde{u}_{x} \frac{\partial \Omega}{\partial \tilde{x}}+u_{\sigma} \frac{\partial \Omega}{\partial \sigma}=\frac{1}{2} \frac{\partial^{2} \Omega}{\partial \tilde{x}^{2}} .
$$

Equation (23) is a parabolic partial differential equation, so we need an initial condition for the variable $\sigma$ and two boundary conditions for the variable $\tilde{x}$. The initial condition can be given by the vorticity distribution $\left[\Omega=\Omega_{0}(\tilde{x})\right]$ at a certain point $\sigma=\sigma_{0}$, which is outside the bubble. The boundary conditions in $\tilde{x}$ are quite obvious. At the equatorial plane outside the bubble $\left(\tilde{x}=0, \sigma>\sigma_{0}\right)$, the vorticity from the two sides of the bubble is cancelled because of the symmetry of the flow, and $\Omega=0$. The other condition is that the vorticity vanishes at the outer edge of the wake region, i.e., $\Omega \rightarrow 0$ as $\tilde{x} \rightarrow \infty$.

To make the problem simpler, let us assume that $\sigma_{0}=O(1)$, but is sufficiently large, so that the velocity field in the wake region can be approximated by the undisturbed biaxial straining flow. This assumes implicitly that the vorticity in the wake region is small enough so that the velocity perturbation, resulting from vorticity, is negligible in the wake region. This assumption is valid if the vorticity in the wake is $O(1)$ at any $\sigma$. The validity of the assumption will be examined a posteriori. The undisturbed velocity field is given by

$$
\tilde{u}_{x}=-\tilde{x}, \quad u_{\sigma}=\frac{1}{2} \sigma .
$$

Now, the governing equation and boundary conditions for the vorticity distribution in the wake are

$$
\frac{\sigma}{2} \frac{\partial \Omega}{\partial \sigma}=\tilde{x} \frac{\partial \Omega}{\partial \tilde{x}}+\frac{1}{2} \frac{\partial^{2} \Omega}{\partial \tilde{x}^{2}},
$$

with

$$
\begin{aligned}
& \Omega=\Omega_{0}(\tilde{x}), \text { at } \sigma=\sigma_{0} \text {, } \\
& \Omega=0, \quad \text { at } \tilde{x}=0 \quad\left(\sigma \geqslant \sigma_{0}\right) \text {, } \\
& \Omega \rightarrow 0, \quad \text { as } \tilde{x} \rightarrow \infty \quad\left(\sigma>\sigma_{0}\right) .
\end{aligned}
$$

The solution of (24) can be easily obtained by separation of variables, and is given as

$$
\Omega=\sum_{k=1}^{\infty} C_{k} e^{-\dot{x}^{2}} H_{2 k-1}(\tilde{x}) \sigma^{-4 k},
$$

where $H_{2 k-1}(\tilde{x})$ is the $(2 k-1)$ st Hermite polynomial and the coefficient $C_{k}$ is given by

$$
C_{k}=\frac{\sigma_{0}^{4 k}}{2^{2 k-2} \sqrt{\pi}(2 k-1) !} \int_{0}^{\infty} \Omega_{0}(\tilde{x}) H_{2 k-1}(\tilde{x}) d \tilde{x} .
$$

Since $\Omega=\omega / \sigma$, the final expression for $\omega$ is

$$
\omega=\sum_{k=1}^{\infty} C_{k} e^{-\tilde{x}^{2}} H_{2 k-1}(\tilde{x}) \sigma^{1-4 k}
$$

The primary and important conclusion we can draw from the result (26) is that although vorticity can grow locally for finite $\sigma$ because of the vortex stretching mechanism, it eventually vanishes because of diffusion as $\sigma \rightarrow \infty$. Physically, the equatorial plane plays the role of a vorticity sink, because $\omega=0$ there as a result of the exact cancellation of vorticity of opposite signs from the two halves of the bubble. Therefore the effectiveness of diffusion for removal of vorticity requires that a large gradient of vorticity be maintained near the equatorial plane. In the biaxial straining flow, such a large gradient is achieved by convection of vorticity with the velocity component, $\tilde{u}_{x}=-\tilde{x}$. This convection effect is sufficient to maintain a large gradient of vorticity close to the vorticity sink (note that the vorticity has a maximum at a certain finite position $\tilde{x}$ for all $\left.\sigma_{0}<\sigma<\infty\right)$. It should be noted that Eq. (26) gives a theoretical basis for using the farfield boundary condition for vorticity, $\omega \rightarrow 0$ as $\sqrt{x^{2}+\sigma^{2}}$ $\rightarrow \infty$, for the numerical analysis in the following sections.

\section{PROBLEM FORMULATION FOR NUMERICAL ANALYSIS}

In Sec. III, we have discussed some preliminary analytical results that will play an important role in understanding the deformation of a bubble in a biaxial straining flow. However, these analyses have been mainly limited to small deformations (i.e., $W<1$ ), the exception being the invariance of steady bubble shapes to changes of flow direction in the potential flow limit. To complete our investigation, we need to examine finite deformation effects. Thus in the following sections, we discuss numerical results for steady and unsteady (finite-amplitude) bubble deformation in a biaxial straining flow for $0<R<400$, and for the potential flow limit, $R=\infty$.

In the numerical analysis that follows, we have used the numerical scheme for unsteady free-boundary problems that was developed in I, except for modifications in the difference scheme for the vorticity transport equation, which will be discussed later. Therefore, in this section, we present only the minimum description necessary for the present work.

All of the computations are performed on a time-dependent (or time-independent, in the case of steady-state analysis) boundary-fitted orthogonal coordinate system $(\xi, \eta)$ as described in $I$. Thus the boundary coordinate at any instant $t$ is connected with the common cylindrical coordinate $(x, \sigma)$ (with the axis of symmetry being the $x$ axis) via a pair of mapping functions $x(\xi, \eta, t)$ and $\sigma(\xi, \eta, t)$, which satisfy the covariant Laplace equations 


$$
\begin{aligned}
& \frac{\partial}{\partial \xi}\left(f \frac{\partial x}{\partial \xi}\right)+\frac{\partial}{\partial \eta}\left(\frac{1}{f} \frac{\partial x}{\partial \eta}\right)=0 \\
& \frac{\partial}{\xi}\left(f \frac{\partial \sigma}{\partial \xi}\right)+\frac{\partial}{\partial \eta}\left(\frac{1}{f} \frac{\partial \sigma}{\partial \eta}\right)=0 .
\end{aligned}
$$

Here, the function $f(\xi, \eta)$ is the so-called distortion function representing the ratio $h_{n} / h_{\xi}$ of scale factors $\left[h_{\eta} \equiv\left(g_{\eta \eta}\right)^{1 / 2}\right.$, $\left.h_{\xi} \equiv\left(g_{\xi \xi}\right)^{1 / 2}\right]$ for the boundary-fitted coordinate system.

The fluid mechanics part of the problem is to obtain solutions of the Navier-Stokes equations using a finite-difference approximation in the boundary-fitted $(\xi, \eta)$ coordinate domain. With axisymmetry assumed, the NavierStokes equations are most conveniently expressed in terms of the streamfunction $\psi$ and vorticity $\omega$ in the form

$$
\begin{aligned}
& \frac{R}{2}\left[\left(\frac{\partial \omega}{\partial t}\right)_{x, \sigma}+\frac{1}{h_{\eta} h_{\xi}}\right. \\
& \left.\quad \times\left(\frac{\partial \psi}{\partial \xi} \frac{\partial}{\partial \eta}\left(\frac{\omega}{\sigma}\right)-\frac{\partial \psi}{\partial \eta} \frac{\partial}{\partial \xi}\left(\frac{\omega}{\sigma}\right)\right)\right]=L^{2}(\omega \sigma),
\end{aligned}
$$

$L^{2} \psi+\omega=0$,

where $(\partial \omega / \partial t)_{x, \sigma}$ is calculated according to the transformation

$$
\begin{aligned}
\left(\frac{\partial w}{\partial t}\right)_{x, \sigma}= & \left(\frac{\partial w}{\partial t}\right)_{\xi, \eta}+\frac{1}{h_{\eta} h_{\xi}}\left(\frac{\partial w}{\partial \xi} \frac{\partial \sigma}{\partial \eta}-\frac{\partial w}{\partial \eta} \frac{\partial \sigma}{\partial \xi}\right)\left(\frac{\partial x}{\partial t}\right)_{\xi, \eta} \\
& -\frac{1}{h_{\eta} h_{\xi}}\left(\frac{\partial \omega}{\partial \xi} \frac{\partial x}{\partial \eta}-\frac{\partial \omega}{\partial \eta} \frac{\partial x}{\partial \xi}\right)\left(\frac{\partial \sigma}{\partial t}\right)_{\xi, \eta}
\end{aligned}
$$

and

$$
L^{2} \equiv \frac{1}{h_{\eta} h_{\xi}}\left(\frac{\partial}{\partial \xi}\left(\frac{f}{\sigma} \frac{\partial}{\partial \xi}\right)+\frac{\partial}{\partial \eta}\left(\frac{1}{f \sigma} \frac{\partial}{\partial \eta}\right)\right) .
$$

The streamfunction $\psi$ in (28) and (29) is defined, so as to satisfy

$$
u_{\xi}=-\frac{1}{\sigma h_{\eta}} \frac{\partial \psi}{\partial \eta}, \quad u_{\eta}=\frac{1}{\sigma h_{\xi}} \frac{\partial \psi}{\partial \xi} .
$$

We assume, for convenience, that the coordinate mapping is defined with $\xi=1$ corresponding to the interface (Fig. 3), where $\eta=0$ and $\eta=1$ are the symmetry axes. The boundary conditions at the symmetry axes are

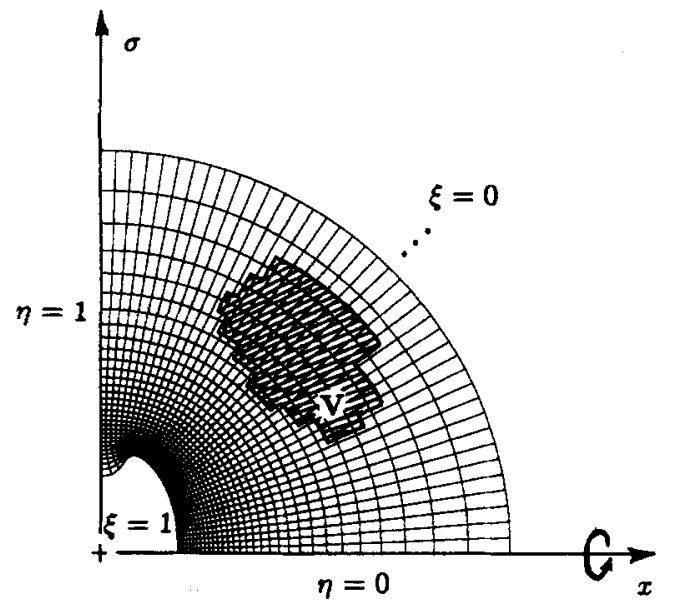

FIG. 3. A boundary-fitted coordinate system and an arbitrary axisymmetric closed volume $V$ in the flow.

$$
\psi=0, \omega=0, \text { at } \eta=0,1 \text {. }
$$

At the gas-liquid interface $(\xi=1)$ we require

$\psi=\int_{0}^{\eta}\left(-u_{\xi}\right) \sigma h_{\eta} d \eta=\int_{0}^{\eta}\left(-\frac{1}{|\nabla F|} \frac{\partial F}{\partial t}\right) \sigma h_{\eta} d \eta$,

corresponding to the kinematic condition. In (31), $F$ is a function that describes the bubble shape as $F(x, t)=0$ and $u_{\xi}$ is the inward normal velocity. In addition, the vorticity at the bubble surface is given by

$$
\omega=\frac{2}{h_{\eta}} \frac{\partial u_{\xi}}{\partial \eta}+2 \kappa_{(\eta)} u_{\eta},
$$

corresponding to the condition of zero tangential stress (where $\kappa_{(\eta)}$ is the normal curvature of the interface in the $\eta$ direction and $u_{\eta}$ is the tangential velocity). Finally, the normal stress contributions resulting from pressure and viscous forces, on the one hand, and the capillary force, on the other, are required to balance

$$
\tau_{\xi \xi}-(4 / W)\left(\kappa_{(\eta)}+\kappa_{(\phi)}\right)=0
$$

In (33), $\kappa_{(\phi)}$ is the normal curvature in the $\phi$ direction, $W$ is the (dimensionless) Weber number, and $\tau_{\xi \xi}$ is the total normal stress, which includes both static and dynamic pressure and viscous stress contributions.

The far-field boundary condition for the biaxial straining flow is given as

$$
\psi \sim-\frac{1}{2} x \sigma^{2}, \quad \omega \rightarrow 0, \quad \text { as } \xi \rightarrow 0\left(\sqrt{x^{2}+\sigma^{2}} \rightarrow \infty\right) .
$$

Since the streamfunction tends to $-\infty$ as $\xi \rightarrow 0$, a new unknown streamfunction $\psi^{*}$ is introduced, which is bounded (see I and Ryskin and Leal ${ }^{16}$ ),

$$
\psi^{*} \equiv \psi+\frac{1}{2} x \sigma^{2}\left(1-\xi^{5}\right) \text {, }
$$

where $-\frac{1}{2} x \sigma^{2}\left(1-\xi^{5}\right)$ is subtracted instead of $-\frac{1}{2} x \sigma^{2}$, in order to have a simple boundary condition at the bubble surface $(\xi=1)$ and $\psi^{*} \equiv 0$ for the limiting case of potential flow around a sphere.

\section{NUMERICAL SCHEME}

The problem, from a numerical point of view, is to solve simultaneously the governing differential equations (27)(29), subject to boundary conditions (30)-(34), as indicated above. In the present analysis, the transient algorithm described in I has been used with only a modification in the difference scheme for the vorticity equation as described below (note that the transient algorithm given in I with the time step $\Delta t=\infty$ degenerates to the steady-state algorithm of Ryskin and Leal ${ }^{16}$ ).

In I and in the paper by Ryskin and Leal, a nonconservative difference scheme was used for the vorticity equation. This led to a highly stable solution algorithm for the uniaxial flow problem considered in I, and a scheme that was stabilized up to moderately large Reynolds numbers, $O(200)$, in the buoyancy-driven bubble translation problem that was considered by Ryskin and Leal. ${ }^{17}$ However, in the present biaxial flow problem, we have found that this nonconservative difference scheme is unconditionally unstable for $R>O(5)$. It appears to us, on the basis of the stability analy- 
sis presented below, and other factors, that there is a general tendency for the nonconservative difference formulation to be unstable at moderate or large Reynolds number, which is realized to a greater or lesser degree, depending upon the nature of the flow. In particular, for axisymmetric flows with converging streamlines in the streamwise direction, such as the uniaxial flow problem considered in I, any tendency to generate disturbance vorticity in the azimuthal direction (all that is possible with the velocity field restricted to axisymmetry) is counteracted to a larger extent by the contraction of vortex lines. On the other hand, for axisymmetric flows with streamlines that diverge in the streamwise direction, such as the biaxial flow considered here, any disturbance vorticity is enhanced via vortex stretching and any natural tendency to inhibit instability is lost. Finally, flows such as the streaming motion past a bubble where streamlines first diverge (locally) over the front half of the body and then converge at the back, will tend by this qualitative picture, to be somewhere between these extremes, insofar as inhibition of numerical instability is concerned.

The natural tendency of some flows to be more susceptible than others to numerical instability cannot be changed, but the intrinsic stability of the numerical scheme can be influenced by the choice of the difference formulation. In the present work, we therefore follow the recommendation of Roache, ${ }^{18}$ and utilize a conservative formulation for the discretized vorticity transport equation. As we shall see, from both a stability analysis and the actual performance of the resulting numerical algorithm, the modified finite-difference scheme is stable for both uniaxial and biaxial straining flows up to of at least $R=400$. In addition, preliminary comparisons with the calculations of Ryskin and Leal, for the rising bubble problem indicate that the conservative formulation of the vorticity transport equation substantially improves stability in that case also.

In the remainder of this section, we first discuss the stability of the nonconservative difference scheme of Ryskin and Leal (also used in I), and then contrast this with the conservative difference scheme that was used for the calculations that are reported in the next section. We shall see that the stability analyses of the two difference schemes largely corroborate the qualitative statements of the preceding paragraphs.

\section{A. Nonconservative difference scheme for the vorticity equation}

We are concerned with both unsteady- and steady-state numerical solutions. Nevertheless, for convenience, we restrict our discussion in this and in Sec. V B to the steadystate algorithm. A discussion of the stability for the unsteady algorithm will be essentially the same, with only minor modifications.

Let us start with the steady-state vorticity transport equation in the form used by Ryskin and Leal, ${ }^{16}$

$$
\mathbf{u} \cdot \nabla \boldsymbol{\omega}-\boldsymbol{\omega} \cdot \boldsymbol{\nabla u}=(2 / R) \nabla^{2} \omega .
$$

For axisymmetric problems, we have $\omega=(0,0, \omega)$, $\mathbf{u}=\left(u_{\xi}, u_{\eta}, 0\right)$, and

$$
\mathbf{e}^{\phi} \cdot \nabla \mathbf{u}=\left[\frac{1}{\sigma} \frac{\partial u_{\xi}}{\partial \phi}, \frac{1}{\sigma} \frac{\partial u_{\eta}}{\partial \phi}, \frac{1}{\sigma}\left(\frac{u_{\xi}}{h_{\xi}} \frac{\partial \sigma}{\partial \xi}+\frac{u_{\eta}}{h_{\eta}} \frac{\partial \sigma}{\partial \eta}\right)\right]
$$

in the $(\xi, \eta, \phi)$ coordinate system. Thus the equation for $\omega$ can be expressed in the following form:

$f^{2} \frac{\partial^{2} \omega}{\partial \xi^{2}}+\frac{\partial^{2} \omega}{\partial \eta^{2}}+q_{1} \frac{\partial \omega}{\partial \xi}+q_{2} \frac{\partial \omega}{\partial \eta}+q_{3} \omega+q_{4}=0$,

where

$$
\begin{aligned}
q_{3}= & \frac{R}{2} f h_{\eta} \frac{u_{\xi}}{\sigma} \frac{\partial \sigma}{\partial \xi}+\frac{R}{2} h_{\eta} \frac{u_{\eta}}{\sigma} \frac{\partial \sigma}{\partial \eta} \\
& -\left(\frac{f}{\sigma} \frac{\partial \sigma}{\partial \xi}\right)^{2}-\left(\frac{1}{\sigma} \frac{\partial \sigma}{\partial \eta}\right)^{2} \\
= & q_{3}^{\text {st }}-\left(\frac{f}{\sigma} \frac{\partial \sigma}{\partial \xi}\right)^{2}-\left(\frac{1}{\sigma} \frac{\partial \sigma}{\partial \eta}\right)^{2} .
\end{aligned}
$$

The other coefficients in (36) are not given explicitly, because only $q_{3}$ is required in the following discussion. The term $q_{3}^{\text {st }} \omega$ corresponds to vorticity enhancement through stretching in the $\phi$ direction in a biaxial flow and, as we shall see, is responsible for numerical instability in a nonconservative formulation. The last two terms are curvature contributions from $\nabla^{2} \omega$ and act to stabilize the numerical scheme.

Now, let us consider the simplest model problem of an axisymmetric straining flow around a spherical bubble. For a spherical bubble, if we take the distortion factor $f=\pi \xi / 2$, then the coordinate transformation of Eq. (27) is given by $x=\xi^{-1} \cos [(\pi / 2) \eta], \quad \sigma=\xi^{-1} \sin [(\pi / 2) / \eta]$, with

$$
h_{\eta}=(\pi / 2) \xi^{-1}, \quad h_{\xi}=\xi^{-2} .
$$

Furthermore, let us assume that the velocity field is given by the potential flow solution around a spherical bubble,

$$
\begin{aligned}
u_{\xi}= & \mp \frac{1}{2 h_{\eta}}\left(1-\xi^{5}\right)\left(2 x \frac{\partial \sigma}{\partial \eta}+\sigma \frac{\partial x}{\partial \eta}\right), \\
u_{\eta}= & \mp \frac{1}{2 h_{\xi}}\left(-2 x\left(1-\xi^{5}\right) \frac{\partial \sigma}{\partial \xi}\right. \\
& \left.-\sigma\left(1-\xi^{5}\right) \frac{\partial x}{\partial \xi}+5 \sigma x \xi^{4}\right),
\end{aligned}
$$

where " - " and " + " of " $\mp$ " refer to the uniaxial and biaxial straining flows, respectively. By substituting (38) and (39) into (37), we get

$$
\begin{aligned}
q_{3}= & \mp\left(\frac{R f}{2}\right)\left[\frac{\pi}{4}\left(\frac{1-\xi^{5}}{\xi^{3}}\right)+\frac{5 \pi}{4} x \xi^{3} \cos \left(\frac{\pi}{2} \eta\right)\right] \\
& -\left(\frac{\pi}{2}\right)^{2}\left(\frac{1}{\sin ^{2}[(\pi / 2) \eta]}\right) \\
\simeq & \mp R\left(\frac{\pi}{4}\right)^{2}\left[\left(\frac{1-\xi^{5}}{\xi^{2}}\right)+5 \xi^{3} \cos ^{2}\left(\frac{\pi}{2} \eta\right)\right],
\end{aligned}
$$$$
\text { for } R \gg 1 \text {. }
$$

Now, let us consider the numerical solution of (36) on an $N \times N$ grid system for the domain $0 \leqslant \xi, \eta \leqslant 1$, with the boundary conditions

$$
\begin{aligned}
& \omega=0, \quad \text { at } \eta=0, \eta=1, \text { and } \xi=0, \\
& \omega=\omega_{0}(\eta), \quad \text { at } \xi=1 .
\end{aligned}
$$


Spatial discretization of (36) with a second-order central difference scheme gives us a system of linear algebraic equations of the form

$$
\mathbf{A w}=\mathbf{b} \text {, }
$$

where

$$
w=\left(\omega_{11}, \ldots, \omega_{1 N}, \ldots, \omega_{N 1}, \ldots, \omega_{N N}\right)
$$

and $\mathrm{A}$ is a $N^{2} \times N^{2}$ matrix. The solution method developed by Ryskin and Leal ${ }^{16}$ used in I for (41) is the alternating direction implicit (ADI) method, which is based on the transformation of (41) into a fictitious initial value problem,

$$
\frac{d \mathbf{w}}{d \tau}=\mathbf{A w}-\mathbf{b} .
$$

In this case, the stability of the numerical scheme is completely determined by the eigenvalues of matrix $\mathbf{A}$. The necessary condition for stability is that the trace of $\mathbf{A}$ should be negative, i.e.,

$$
\sum_{k=1}^{N^{2}} \lambda_{k}=\sum_{k=1}^{N^{2}} a_{k k}<0
$$

otherwise at least one eigenvalue must be positive, implying instability. The diagonal elements of $\mathbf{A}$ can be expressed as

$$
\begin{aligned}
\alpha_{k k} & =-\frac{2}{h^{2}}\left(f_{i j}^{2}+1\right)+q_{3, i j} \\
& \simeq-\frac{2}{h^{2}}\left[\left(\frac{\pi}{2}\right)^{2} \xi_{i j}^{2}+1\right] \\
& \mp R\left(\frac{\pi}{4}\right)^{2}\left[\left(\frac{1-\xi_{i j}^{5}}{\xi_{i j}^{2}}\right)+5 \xi_{i j}^{3} \cos ^{2}\left(\frac{\pi}{2} \eta_{i j}\right)\right],
\end{aligned}
$$

where $h=1 / N, k=(i-1) \times N+j$, and the indices $i$ and $j$ stand for the grid point $\left(\xi_{i j}, \eta_{i j}\right)=(i h, j h)$. The necessary condition for stability is satisfied for all $R$ in the case of uniaxial straining flow, because $a_{k k}<0$ for all $k$, as we can see in (44) (" - " of $\mp$ is for the uniaxial straining flow). However, the necessary condition for stability is violated as $R \rightarrow \infty$ in the case of biaxial straining flow, because

$$
\begin{aligned}
\sum_{k=1}^{N^{2}} a_{k k}= & \sum_{k=1}^{N^{2}}\left\{-\frac{2}{h^{2}}\left[\left(\frac{\pi}{2}\right)^{2} \xi_{i j}^{2}+1\right]\right. \\
& \left.+R\left(\frac{\pi}{4}\right)^{2}\left[\left(\frac{1-\xi_{i j}^{5}}{\xi_{i j}^{2}}\right)+5 \xi_{i j}^{3} \cos ^{2}\left(\frac{\pi}{2} \eta_{i j}\right)\right]\right\} \\
> & -2\left[\left(\frac{\pi}{2}\right)^{2}+1\right] \frac{1}{h^{4}}+R\left(\frac{\pi}{4}\right)^{2} \frac{1}{h^{3}} \\
> & \frac{-14+R h}{2 h^{4}} .
\end{aligned}
$$

Therefore, if $R>14 / h$, the numerical scheme must be unstable. In fact, when a $\mathbf{4 0} \times \mathbf{4 0}$ grid system was used in our numerical computation, the ADI scheme with a nonconservative difference scheme failed to converge for $R>5$ in the case of biaxial straining flow, while convergent solutions were obtained for all $R$ up to the maximum value considered $(R \leqslant 100)$ in the uniaxial straining flow problem.

Now, the question is whether we can avoid this numerical instability while maintaining second-order accuracy in the spatial discretization. The answer is given in Sec. V B.

\section{B. Modified difference scheme for the vorticity transport equation}

Let us begin again with the vorticity transport equation for an axisymmetric problem in the $(\xi, \eta, \phi)$-coordinate system,

$$
\frac{R}{2}\left[\mathbf{u} \cdot \nabla \omega-\left(\frac{\omega}{\sigma} \frac{u_{\xi}}{h_{\xi}} \frac{\partial \sigma}{\partial \xi}+\frac{\omega}{\sigma} \frac{u_{\eta}}{h_{\eta}} \frac{\partial \sigma}{\partial \eta}\right)\right]=L^{2}(\omega \sigma),
$$

where the second term on the left-hand side is still just $\omega \cdot \nabla u$ from (38). To express (46) in a conservative form, we first note

$$
\begin{aligned}
\mathbf{u} \cdot \nabla \omega & =\nabla \cdot(\omega \mathbf{u}) \\
& =\frac{1}{h_{\xi} h_{\eta} \sigma}\left(\frac{\partial}{\partial \xi}\left(h_{\eta} \sigma \omega u_{\xi}\right)+\frac{\partial}{\partial \eta}\left(h_{\xi} \sigma \omega u_{\eta}\right)\right),
\end{aligned}
$$

because of the continuity equation $(\nabla \cdot u=0)$. Hence substituting (47) into (46), and combining the two sets of resulting terms on the left-hand side, we have

$$
\begin{aligned}
\frac{R}{2} \sigma\left[\nabla \cdot\left(\frac{\omega}{\sigma} u\right)\right]= & \frac{R}{2} \frac{1}{h_{\xi} h_{\eta}}\left(\frac{\partial}{\partial \xi}\left(h_{\eta} \omega u_{\xi}\right)\right. \\
& \left.+\frac{\partial}{\partial \eta}\left(h_{\xi} \omega u_{\eta}\right)\right)=L^{2}(\omega \sigma) .
\end{aligned}
$$

Then Eq. (48) can be expressed in the form

$$
\begin{gathered}
f^{2} \frac{\partial^{2} \omega}{\partial \xi^{2}}+\frac{\partial^{2} \omega}{\partial \eta^{2}}+\tilde{q}_{1} \frac{\partial \omega}{\partial \xi}+\tilde{q}_{2} \frac{\partial \omega}{\partial \eta}+\tilde{q}_{3} \omega+\tilde{q}_{5} \\
\times\left(\frac{\partial}{\partial \xi}\left(h_{\eta} \omega u_{\xi}\right)+\frac{\partial}{\partial \eta}\left(h_{\xi} \omega u_{\eta}\right)\right)=0,
\end{gathered}
$$

where

$$
\tilde{q}_{3}=-\left[\left(\frac{f}{\sigma} \frac{\partial \sigma}{\partial \xi}\right)^{2}+\left(\frac{1}{\sigma} \frac{\partial \sigma}{\partial \eta}\right)^{2}\right]
$$

and

$$
\tilde{q}_{5}=-(R / 2) f .
$$

In discretizing (49), we apply the central difference form directly to the last two terms. For example,

$$
\frac{\partial}{\partial \xi}\left(h_{\eta} \omega u_{\xi}\right)=\frac{\left(h_{\eta} \omega u_{\xi}\right)_{i+1, j}-\left(h_{\eta} \omega u_{\xi}\right)_{i-1, j}}{2 h}+O\left(h^{2}\right) \text {. }
$$

Here we must note that the difference formula (50) requires the boundary conditions at $\xi=0\left(\sqrt{\sigma^{2}+x^{2}}=\infty\right)$ in the form of $h_{\eta} \omega u_{\xi}$ as well as $\omega$ itself. From the earlier parts of this paper, we see that $\omega=O\left(\sigma^{-3}\right)=O\left(\xi^{3}\right)$ [Eq. (26)] and $h_{\eta} u_{\xi}=O\left(\xi^{-2}\right)$ [Eq. (39)] in the limit $\xi \rightarrow 0$. Therefore, we have $h_{\eta} \omega u_{\xi}=0$ as well as $\omega=0$ at $\xi=0$.

Now, let us consider the discretized equation of the fictitious initial value problem for (49) in the form

$$
\frac{d \mathbf{w}}{d \tau}=\tilde{\mathbf{A}} \mathbf{w}-\tilde{\mathbf{b}} \text {. }
$$

In (51), the diagonal element of $\widetilde{\mathbf{A}}$ is given as

$$
\tilde{a}_{k k}=-\left(2 / h^{2}\right)\left(f_{i j}^{2}+1\right)+\tilde{q}_{3, i j}<0, \text { for all } k,
$$
where $h=1 / N, k=(i-1) \times N+j$. Therefore the neces- 
sary condition for stability is automatically satisfied for all types of axisymmetric flow and for all Reynolds numbers, while second-order accuracy is preserved, as we can see in (50).

Finally, before proceeding to discuss numerical results, it is of interest to discuss the sense in which the difference scheme in $(50)$ conserves vorticity. To do that we consider the term

$$
\boldsymbol{\nabla} \cdot\left(\frac{\omega}{\sigma} \mathbf{u}\right)=\frac{1}{h_{\xi} h_{\eta} \sigma}\left(\frac{\partial}{\partial \xi}\left(h_{\eta} \omega u_{\xi}\right)+\frac{\partial}{\partial \eta}\left(h_{\xi} \omega u_{\eta}\right)\right),
$$

which is in the first term of (48). The differential term on the left is conservative in the sense that

$$
\int_{v} \boldsymbol{\nabla} \cdot\left(\frac{\omega}{\sigma} \mathbf{u}\right) d V=\int_{s}\left(\frac{\omega}{\sigma} \mathbf{u}\right) \cdot \mathbf{n} d S,
$$

where $V$ is an arbitrary, axisymmetric closed volume in the flow region and $S$ is the closed surface of $V$.

We will show that the discretized approximation of the right-hand side, based on (50), satisfies the same conservation relationship. Consider any domain, such as the shaded region in Fig. 3, for which the grid system is given as

$$
\begin{array}{ll}
n(j)-\frac{1}{2} \leqslant i \leqslant N(j)+\frac{1}{2}, & \text { where } n \leqslant n(j) \leqslant N(j) \leqslant N, \\
m(i)-\frac{1}{2} \leqslant j \leqslant M(i)+\frac{1}{2}, & \text { where } m \leqslant m(i) \leqslant M(i) \leqslant M .
\end{array}
$$

Then,

$$
\begin{aligned}
\int_{v} \boldsymbol{\nabla} \cdot( & \left.\frac{\omega}{\sigma} \mathbf{u}\right) d V \\
= & 2 \pi \int_{R}\left(\frac{\partial}{\partial \xi}\left(h_{\eta} \omega u_{\xi}\right)+\frac{\partial}{\partial \eta}\left(h_{\xi} \omega u_{\eta}\right)\right) d \xi d \eta \\
= & 2 \pi \sum_{j=m}^{M} \sum_{i=n(j)}^{N(j)}\left(\frac{\left(h_{\eta} \omega u_{\xi}\right)_{i+1, j}-\left(h_{\eta} \omega u_{\xi}\right)_{i-1, j}}{2 h}\right) \cdot h^{2}+2 \pi \sum_{i=n}^{N} \sum_{j=m(i)}^{M(i)}\left(\frac{\left(h_{\xi} \omega u_{\eta}\right)_{i, j+1}-\left(h_{\xi} \omega u_{\eta}\right)_{i, j-1}}{2 h}\right) \cdot h^{2} \\
= & 2 \pi \sum_{j=m}^{M}\left(\frac{\left(h_{\eta} \omega u_{\xi}\right)_{N(j)+1, j}+\left(h_{\eta} \omega u_{\xi}\right)_{N(j), j}}{2}-\frac{\left(h_{\eta} \omega u_{\xi}\right)_{n(j)-1, j}+\left(h_{\eta} \omega u_{\xi}\right)_{n(j), j}}{2}\right) \cdot h \\
& +2 \pi \sum_{i=n}^{N}\left(\frac{\left(h_{\xi} \omega u_{\eta}\right)_{i, M(i)+1}+\left(h_{\xi} \omega u_{\eta}\right)_{i, M(i)}}{2}-\frac{\left(h_{\xi} \omega u_{\eta}\right)_{i, m(i)-1}+\left(h_{\xi} \omega u_{\eta}\right)_{i, m(i)}}{2}\right) \cdot h \\
= & 2 \pi \sum_{j=m}^{M}\left[\left(h_{\eta} \omega u_{\xi}\right)_{N(j)+1 / 2, j}-\left(h_{\eta} \omega u_{\xi}\right)_{n(j)-1 / 2, j}\right] \cdot h+2 \pi \sum_{i=n}^{N}\left[\left(h_{\xi} \omega u_{\eta}\right)_{i, M(i)}+1 / 2-\left(h_{\xi} \omega u_{\eta}\right)_{i, m(i)-1 / 2}\right] \cdot h \\
= & \int_{s}\left(\frac{\omega}{\sigma} \mathbf{u}\right) \cdot \mathbf{n} d S .
\end{aligned}
$$

Therefore the difference scheme of $(50)$ satisfies the conservative property. One consequence of this is that the vorticity distribution in an axisymmetric, steady inviscid flow must satisfy the so-called Kelvin's circulation theorem. Since $\boldsymbol{\nabla} \cdot[(\omega / \sigma) \mathbf{u}]=0$, we have

$$
\int_{s}\left(\frac{\omega}{\sigma} \mathbf{u}\right) \cdot \mathbf{n} d S=0 \text {. }
$$

We also know that

$$
\int_{s} \mathbf{u} \cdot \mathbf{n} d S=0
$$

is true, since we have used the streamfunction and vorticity formulation in the numerical analysis. The only way to satisfy both conditions simultaneously is that $\omega / \sigma=$ const along a streamline, which is, in fact, a steady-state version of Kelvin's circulation theorem. Therefore the conservative difference scheme in the present study is guaranteed to satisfy Kelvin's circulation theorem. Further, as we have seen, it is inherently stable in either uniaxial or biaxial straining flows.

In the following sections, the numerical results obtained via the modified scheme will be presented.

\section{STEADY-STATE NUMERICAL SOLUTIONS}

\section{A. Results}

We have done computations for $R=0,1,10,50,100$, 200,400 , and for potential flow, gradually increasing $W$ in each case. For all $R \leqslant 200$, this procedure was continued until a highly deformed shape was reached that required more resolution than was available with the $40 \times 40$ grid system used here in conjunction with the coordinate mapping scheme. In the potential flow limit, on the other hand, $W$ was increased until a value of 2.7 was reached, beyond which additional steady-state solutions could not be achieved. The procedure for $R=400$ was different, and will be discussed below. The computed bubble shapes for various Reynolds numbers and Weber numbers are shown in Fig. 4, and the streamlines and vorticity contours for selected Reynolds numbers and Weber numbers are also shown in Fig. 5. The most obvious difference between the biaxial flow problem considered here, and the uniaxial flow problem considered earlier in I and by Ryskin and Leal ${ }^{6}$ is that steady solutions were obtained up to quite large Weber numbers for all finite $\boldsymbol{R}$. Indeed, in the present case, no limit point was found over the whole range of $W$ considered for any $R \leqslant 200$.

For $R=400$, however, the behavior was different, with an apparent limit point occurring around $W \sim 6$ for the branch of steady solutions that begins at $W=0$. The use of solid and dashed lines to outline bubble shapes at $R=400$ is intended to indicate that the solutions lie on two separate branches. The solutions at $W=2,4$, and 5 were obtained, starting from a sphere at $W=0$, and gradually incrementing $W$. The solution at $W=5$ was obtained without any particular difficulty. However, when $W$ was incremented upward to 


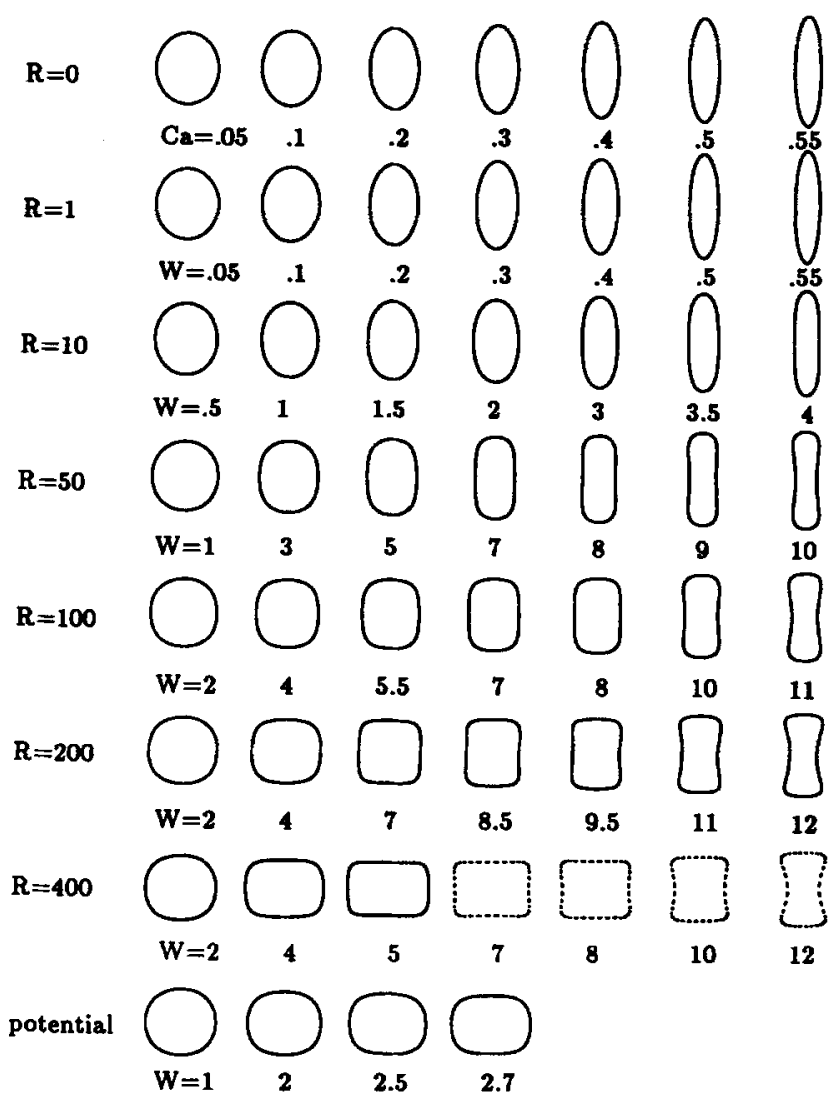

FIG. 4. The steady-state shapes of a bubble in a biaxial straining flow for various Reynolds and Weber numbers (in all figures, the horizontal axis is the axis of symmetry and the vertical axis is the equatorial plane).

$W=6$, no steady solution could be obtained (therefore a limit point for this branch is expected between $W=5$ and $W=6$ ). On the other hand, the solutions for $W=7,8,10$, and 12 were obtained, starting with the solution for $R=200 / W=12$ as an initial guess of the shape for $R=400 / W=12$, and then incrementing $W$ downward to $W=7$. Again, no particular difficulty was experienced in obtaining the solution at $W=7$, but no steady solution could be found on this branch when $W$ was decreased to $W=6$ (again, another limit point for this second branch is expected between $W=6$ and $W=7$ ). Of course, the fact that we could not obtain a converged, steady solution for $W=6$ does not prove that such a solution does not exist. However, we see no reason to believe that there is anything intrinsically more difficult about the numerical problem at $W=6$, than for $W=5$ or $W=7$. Another factor that seems to corroborate our suggestion of a limit point for both solution branches at $W \sim 6$ for $R=400$, is the observation that any added increment in $W$ above $W=5$ or below $W=7$ tends to produce a shape with a waist. We have already noted that all of our earlier studies of bubble or drop deformation at both large and small $R$ suggested that a limit for steady solutions occurs when (if) the bubble developed a "waist." In spite of our confidence in these arguments, however, we are currently exploring the solution behavior between $W=5$ and $W=7$ in more detail, using a full Newton's scheme ${ }^{19}$ to determine whether true limit points exist or not. Unfortunately, we were not able to carry the present solution

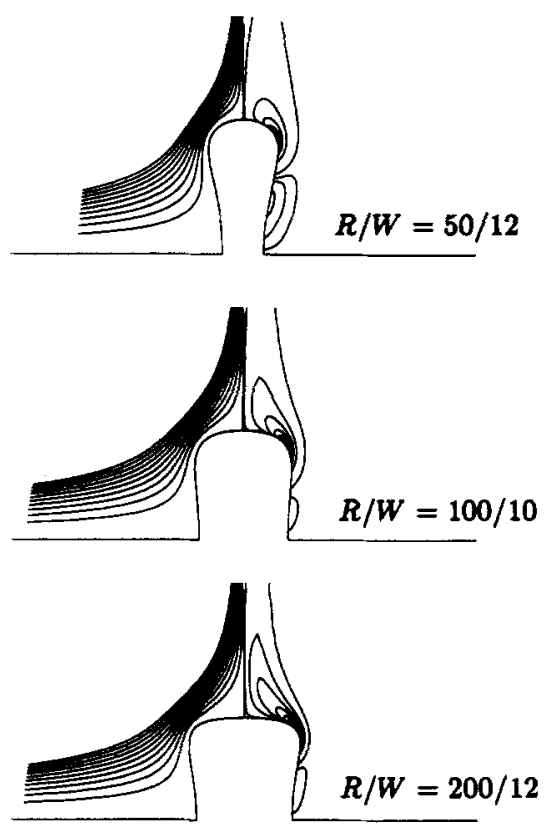

(a)
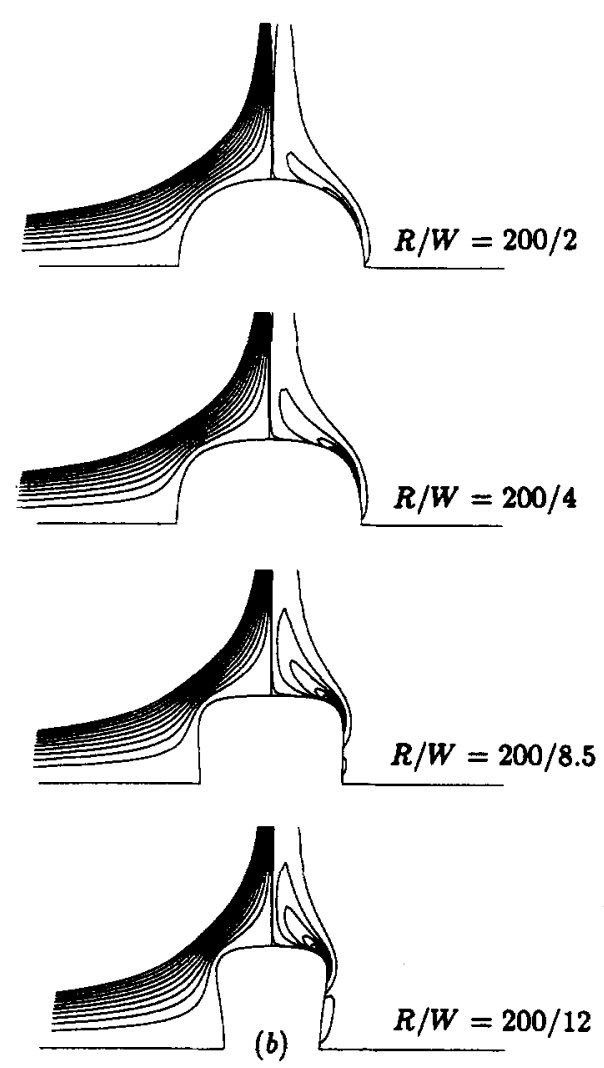

FIG. 5. The streamlines and vorticity contours for various Reynolds and Weber numbers (in all figures, the horizontal axis is the axis of symmetry and the vertical axis is the equatorial plane).

scheme above $R=400$, due to a lack of available computing resources (higher $R$ would require a finer grid and more computing effort because of impending instabilities in the scheme at higher $R$ ).

The fact that no limit point was found numerically for the smaller values of $R<200$, is apparently a consequence of the fact that the bubble is either oblate for all $W$, or else 
undergoes a transition from prolate to oblate shapes before reaching a degree of elongation along the symmetry axis that leads to a "waist" (which we believe to correspond to a limit point). For $R=400$, on the other hand, a critical degree of elongation along the symmetry axis is apparently reached before the bubble can begin to contract toward less elongated shapes. Our numerical evidence suggests that the limit point for steady solutions lies between $W=5$ and $W=6$ when the Weber number is increased from below, and between $W=6$ and $W=7$ when the Weber number is decreased from above. Thus it is our current belief that the two sets of solutions shown in Fig. 4 for $R=400$ represent two distinct (nonintersecting) solution branches. We will address two obvious questions about these statements shortly: first, why does the transition from prolate to oblate shapes take place (see, also, Sec. III B) and, second, why does the oblate shape exhibit steady solutions over a much wider range of $W$ ? The existence of oblate solutions up to relatively large $W$ does not imply, of course, that a limit point may not appear at still larger values of $W$. However, if so, it must occur for $W$ considerably in excess of $O(10)$, and examination of Fig. 4 suggests that it would be a consequence of reaching a configuration where the normal stress balance can only be satisfied by an unacceptable shape in which the two sides of the bubble intersect.

We will discuss the details of the steady-state solutions shown in Figs. 4 and 5, including an attempt to answer the two questions that were posed above. First, however, we attempt to conclude the present discussion by summarizing the conditions in the $R-W$ parameter space for existence of steady solutions for the bubble shape, including both uniaxial and biaxial extensional flows. For the uniaxial extensional flow, the numerical solutions of I and Ryskin and Leal ${ }^{6}$ indicate that there is a critical $W_{c}(R)$, for all $R \geqslant 10$, which is a limit point for the branch of steady solutions that begins with a sphere at $W=0$. Further, this limit point is a monotonically increasing function of $R$ and coverges smoothly to $W_{c}(\infty)$, which is the potential flow limit. Physically, the limit point seems to correspond to the first appearance of a "waist" in the slightly prolate shapes that are obtained, though it is not obvious to us that this should necessarily be the case. We shall discuss this point in more detail shortly. Finally, although we have not made an exhaustive search, we believe it likely that the steady solution branch that we have obtained numerically for the uniaxial flow problem is unique; i.e., no other stable steady solutions exist for larger values of $W$. The situation for biaxial extensional flow is quite different. In the potential flow limit, the behavior is identical to the uniaxial flow case with a limit point appearing at $W_{c} \sim 2.7$, when the bubble shape approaches a prolate configuration with a waist. For $R=400$, this same behavior is found at $W_{c} \sim 6$ if $W$ is increased from zero, and, presumably, for $400<R<\infty$, we would also obtain a critical $W_{c}$, corresponding to prolate shapes with a "waist," with a gradual transition to $W_{c}(\infty)$ from above. It may be noted that the approach of $W_{c}(R)$ to $W_{c}(\infty)$ from below for the uniaxial extension flow, and from above for the biaxial extensional flow is qualitatively consistent with the small deformation analysis of Sec. III B, which shows that the bubble will be more prolate at finite $R$ in the uniaxial case, but less prolate at finite $R$ in the biaxial flow. For $R \leqslant 200$, in the biaxial flow problem, this limit point corresponding to prolate shapes with a waist no longer exists because the bubble shapes transform to a fundamentally different (and stable) oblate form at relatively small values of $W$. For these cases, it appears likely, from the trends exhibited in Fig. 4, that steady solutions will exist all the way to a critical $W>O(10)$, where the normal stress balance would require the two sides of the bubble to intersect. Finally, even for $R>200$, it appears from the solutions obtained at $R=400$ that indented oblate shapes can appear as steady solutions for large $W$. However, above some critical $R$, this second branch of the steady solutions is apparently distinct from the branch that begins with a sphere at $W=0$.

\section{B. Discussion}

In the remainder of this section, we present a more detailed discussion of the results shown in Figs. 4 and 5, including an explanation of the transition to oblate shapes at finite $R$, and a discussion of the observed stability of oblate shapes for relatively large values of $W$. We begin with the results at low Reynolds numbers.

At very low Reynolds numbers, represented in Fig. 4 by $R=0$ and $R=1$, there is a general trend for a bubble to be flattened monotonically to an oblate shape with an increase in the capillary number, $\mathrm{Ca}=\mu E a / \gamma=W / R($ if $R \neq 0)$. As a consequence of this deformation of shape, the curvature of the interface becomes very large at the edge of the bubble, but positive curvature is maintained at the stagnation points on the symmetry axis for all values of $\mathrm{Ca}$ that we have considered. It should be noted that the maximum values of $\mathrm{Ca}$ or $W$ shown in Fig. 4 for the lowest values of $R$ do not represent a limit to the existence of steady solutions. Rather, the computation was simply terminated when the maximum curvature on the rim reached a point where adequate resolution was not possible with the $40 \times 40$ mapped coordinate grid that was being used.

Since no theoretical work is available for this extremely deformed (flattened) bubble, no explicit comparison can be given. Indeed, as stated earlier, we are aware of only one previous analysis of the problem of a bubble in a biaxial straining flow by Frankel and Acrivos. ${ }^{14}$ They computed bubble shapes in the creeping flow limit $(R=0)$ up to an intermediate capillary number $(\mathrm{Ca}=0.2)$ via a semianalytical approach. The results of Frankel and Acrivos for $R=0$ are compared with those of the present work in Fig. 6. Also shown are the numerical results for a uniaxial creeping flow by Youngren and Acrivos ${ }^{4}$ and Ryskin and Leal. ${ }^{6}$ In Fig. 6, the magnitude of deformation is measured in terms of $D=(l-b) /(l+b)$, where $l$ is the radius of the longer axis and $b$ is the radius of the shorter axis.

As the Reynolds number increases, the edge of the bubble at the equatorial plane first becomes more rounded and then flattened at higher Reynolds numbers, and the surface near the stagnation point on the symmetry axis also becomes increasingly flattened until eventually it becomes indented, with negative curvature, for large $W$ and $R>50$. Negative curvatures may also occur at very high $W$ values for lower 


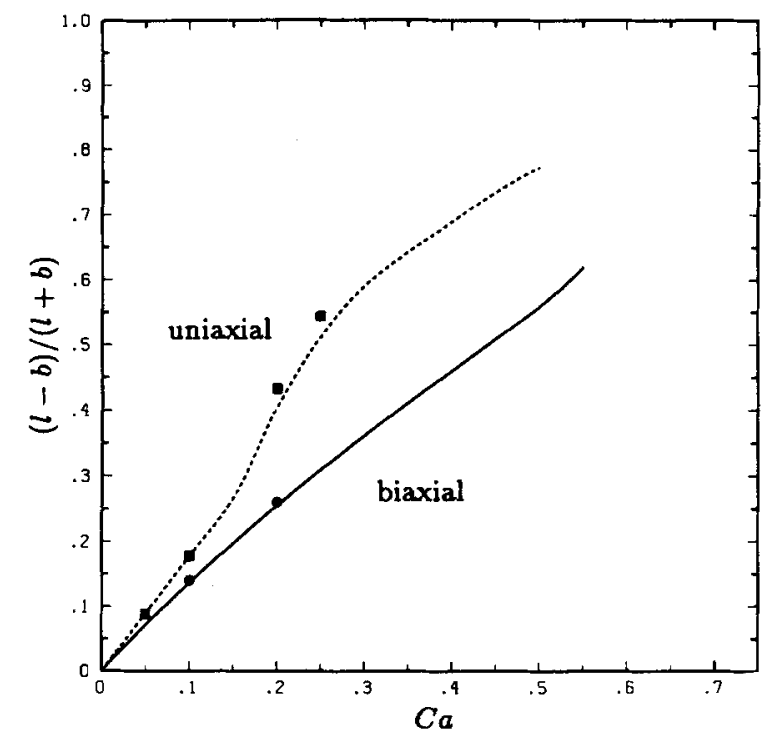

FIG. 6. A comparison of the present results for a bubble in a biaxial straining fow at $\boldsymbol{R}=0$ with the result of Frankel and Acrivos, ${ }^{14}$ and the results for the problem of uniaxial straining flow [-: present result for biaxial flow; : Frankel and Acrivos for biaxial flow: - - -: Youngren and Acrivos ${ }^{4}$ for uniaxial flow; $\square$ : Ryskin and Leal $^{6}(R=0.1)$ for uniaxial flow].

Reynolds numbers, but this is not evident in the present solutions. The existence of negative curvature at the axial stagnation points is not, in itself, particularly surprising. Since the pressure inside the bubble is uniform, the bubble shapes at the higher Reynolds numbers are primarily determined by the dynamic pressure distribution in the external fluid, and this pressure exhibits maximum values at the two stagnation regions where the bubble surface intersects the axis of symmetry and the orthogonal symmetry plane. The bubble responds to these dynamic pressure maxima by being pushed inward to either reduce the positive curvature or yield a region of negative curvature, and thus produce a corresponding increase in the normally directed capillary force.

Of course, the same basic scenario is also relevant to bubble shapes in the uniaxial extensional flow. However, in this case it is the stagnation region at the equatorial plane that is most influenced by the dynamic pressure maxima and the bubble tends toward a slightly elongated shape with a waist. The fundamental difference between the uniaxial and biaxial cases is that no steady solution exists in the uniaxial case with a waist (i.e., with negative curvature at the equatorial plane). Indeed, the numerical solutions of Ryskin and Leal and I show that the dividing point between shapes with positive and negative curvature in the axial direction corresponds to a limit point in Weber number beyond which steady solutions are not possible.

Thus, we see that the primary difference between the uniaxial and biaxial cases is that the dimpled configuration with negative curvature can exist as a steady state in the biaxial flow, whereas the corresponding waisted configuration with negative curvature in the axial direction cannot exist as a steady state in the uniaxial flow. As a consequence, steady solutions can exist to a much higher Weber number in the biaxial flow than in the uniaxial flow. The critical difference between the two cases is the sign of the total curvature in the region of the indentation. In the dimpled configuration, the total curvature is negative, and increases in $W$ can be compensated by an increased degree of dimpling. In the waisted configuration, on the other hand, the situation is more complicated, and an increase in $W$ cannot necessarily be compensated by an increase in the magnitude of indentation at the waist. Indeed, the fact that this does not occur is clearly demonstrated by the observation that the limit point for steady solutions is actually coincident with the initial appearance of a waist!

In the waisted configuration, an increase in the depth of the indentation produces increased negative values of the curvature component

$$
\kappa_{(\eta)}=-\frac{1}{h_{\eta}^{2}} \frac{\partial^{2} \sigma}{\partial \eta^{2}}, \quad \text { at } \eta=1
$$

(as is necessary to balance an increase in the external pressure), but increased positive values of the component,

$$
\kappa_{(\phi)}=1 / \sigma, \text { at } \eta=1 \text {. }
$$

Thus increased indentation of the waist does not necessarily produce a configuration with increased negative curvature, as would be necessary to balance the increased external pressure that is associated with an increase of $W$. This depends on the relative magnitudes of $\kappa_{(\eta)}$ and $\kappa_{(\phi)}$. If the length of the drop is "large" (and the "wavelength" of the indentation is also large), then increased indentation actually yields a more positive value of the total curvature (i.e., $\kappa_{(\eta)}+\kappa_{(\phi)}$ ) and the bubble cannot attain an equilibrium shape for larger $W$, once it reaches a steady shape with $\kappa_{(\eta)}=0$ at the equatorial plane. On the other hand, if the bubble is relatively short when it achieves the incipient waist configuration, then $\left|\kappa_{(\eta)}\right|>\left|\kappa_{(\phi)}\right|$, and we should expect to be able to achieve steady waisted configurations for larger $W$. Apparently, in the parameter ranges considered here, the bubble is always sufficiently long (relative to its cross-sectional radius) when it reaches an incipient waist configuration that no stable "waisted" continuations of shape are possible.

A somewhat different way to understand the difference in stability between dimpled oblate shapes and waisted prolate shapes, is to consider what would happen if the extensional flow were suddenly removed. In the oblate case, the capillary effect produces a maximum in pressure at the dimple, and this tends to drive fluid away from the dimpled region, thus allowing the bubble to return toward a spherical shape. For the prolate case, on the other hand, the tendency toward increased external capillary pressure because of negative $\kappa_{(\eta)}$ can be compensated by the increase in $\kappa_{(\phi)}$. In this case, the capillary pressure contribution resulting from the formation of a waist will be a local minimum instead of maximum, and fluid will be drawn inward toward $\eta=1$, causing the waist to pinch off into a pair of bubbles. The clear implication, in this case, is that the waisted configuration does not provide a mechanism to resist an increase in dynamic pressure (unlike the dimpled configuration), and the Weber number corresponding to incipient formation of a waist must also be a limit for existence of steady solutions.

The present results, at the highest Reynolds numbers, exhibit especially interesting behavior. Specifically, for small 
Weber numbers, the bubble is at first elongated in the axial direction, and only reverts to the flattened pill-like shape for finite $R$ at higher $W$ values. Alternatively, we may note that for any fixed (small) $W$, there is a marginal Reynolds number, as predicted in Sec. III, at which the elongational direction is changed. The estimated marginal Reynolds number in Sec. III was $R_{m}=115$, and it can be seen from Fig. 4 that the numerical solution does change form between $R=100$ and $R=200$. The bubble at $R=100$ (for either $W=2$ or 4 ) is elongated in the direction of the equatorial plane, but the bubble at $R=200$ ( $W=2$ or 4 ) is elongated in the axial direction. This trend toward shapes that are elongated in the axial direction for slightly deformed bubbles is smoothly continued to the potential flow limit for small $W$. However, for $R=200$, the elongation in the direction of the symmetry axis is not maintained as the Weber number increases. Instead, as the curvature at the bubble shoulders is increased, there is an increasing accumulation of vorticity in the vicinity of the equatorial plane and a corresponding decrease in the pressure in this region relative to that on the upstream face of the bubble. This transition is shown in Fig. 7. As a consequence, the bubble surface moves out in the equatorial plane and the bubble becomes increasingly flattened for higher Weber numbers. For $R=400$, on the other hand, the tendency toward elongation in the axial direction is more pronounced, and before the accumulation of vorticity can produce a significant change in shape, the bubble hits a limit point near $W=6$ that is similar in character to the limit behavior at $R=\infty$. As we have already noted, the solutions for $W \geqslant 7$, shown in Fig. 4, are believed by us to lie on a separate solution branch. Although the difference in behavior between the potential flow limit and the present solutions up to $R=200$ might seem quite unexceptional, since $R=200$ is hardly $R=\infty$, the fact is that the corresponding solutions for a bubble in a uniaxial straining flow obtained by Ryskin and Leal ${ }^{6}$ converge smoothly toward the potential flow solutions. At the largest Reynolds number considered by them, $R=100$, the solution is already very similar to the potential flow solution, including the existence of a limit point for steady solutions at $W_{c} \sim 2.2$, which is quite close to the potential flow value, $W_{c} \sim 2.7$. Comparison between the present solutions and those of Ryskin and Leal ${ }^{6}$ shows clearly that there is a fundamental difference in behavior for large, but finite $R$, in spite of the fact that the ultimate limiting behavior in both cases appears to approach the same potential flow limit.

Now, the question is what kind of fundamental difference exists between the uniaxial and biaxial flow cases for $R \neq \infty$, which may explain the differences in behavior for the two cases. The basis of the answer to this question lies in the interaction between the bubble shape, the production of vorticity resulting from the curvature of the bubble surface, and the enhancement of vorticity in the near-wake region downstream of the bubble by vortex stretching. Indeed, for finite $R$ where vorticity can be produced, the fundamental difference between uniaxial and biaxial flow is that the principal axis of elongation in the near wake downstream of the bubble is orthogonal to the vorticity axis for the uniaxial case (so that vorticity only convects and diffuses, but is not enhanced through vortex stretching), while for the biaxial case the elongation direction and the vorticity are parallel so that the vorticity can be increased in magnitude by vortex-line stretching. Thus, all else being equal, the vorticity levels downstream (near the equatorial plane) for the biaxial case should be larger than downstream (along the axis of symmetry) in the uniaxial case. For a given Reynolds number $R$, the magnitude of the vorticity at the bubble surface is proportional to the local curvature, which increases as $W$ increases. In particular, the pressure induced indentation along the symmetry axis, combined with stretching in the equatorial plane, produces shapes with increasing curvature at the shoulder between these two regions, and this, in turn, leads to increasing vorticity production with an increase of $R$, for fixed $W>4$ (or for an increase of $W$ ).

\section{NUMERICALSOLUTIONS FOR UNSTEADY BUBBLE DEFORMATION}

The numerical solutions of Sec. VI show that steadystate solutions exist for large, but finite, $R$ up to very large values of the Weber number. In the present section, we consider transient deformations for both finite $R$ and for the potential flow limit, $R=\infty$. Our goals are twofold: for finite $R$, we show that the steady-state solutions of Sec. VI are stable in the sense that they represent the final steady-state shapes for a wide range of initial shapes; for $R=\infty$, on the other hand, we investigate the transient changes in shape for step changes to both supercritical and subcritical values of $W$. The numerical scheme used to obtain these transient solutions was the same as developed in I, except that we employ the conservative difference scheme for the vorticity transport equation, as discussed in Sec. V.

\section{A. Unsteady deformation in potential flow}

We begin by considering transient shapes for the potential flow limit. It may be recalled from Sec. III, that the oscillation frequencies of the primary deformation mode in an inviscid fluid should be the same in uniaxial and biaxial flows up to $O(W)$. However, no general relationship exists between transient bubble deformation for uniaxial and biaxial flows, and we have thus done two different numerical experiments for the biaxial flow case, in order to complement the transient solutions for uniaxial flow that were reported in I. One is for unsteady deformation following a step increase to a supercritical Weber number, and the other is for oscillatory deformation following a step decrease in $W$ to a subcritical value.

First, we consider the unsteady deformation following a step increase to a supercritical Weber number, $W>W_{c}$. Starting from the steady-state solution for $W=2.7$, the Weber number was increased to 2.9 at $t=0$. In Fig. 8 , the unsteady deformation is shown for $1.7 \leqslant t \leqslant 4.5$. Surprisingly, the bubble initially elongates against the flow direction. However, later, the elongation in that direction is stopped, and the bubble becomes waisted with increasing magnitude for increasing time. In order for a bubble to be elongated against the flow direction, the bubble must do enough work on the fluid to overcome the inertia of the flow. Therefore indefinite elongation against the flow is impossible for this 


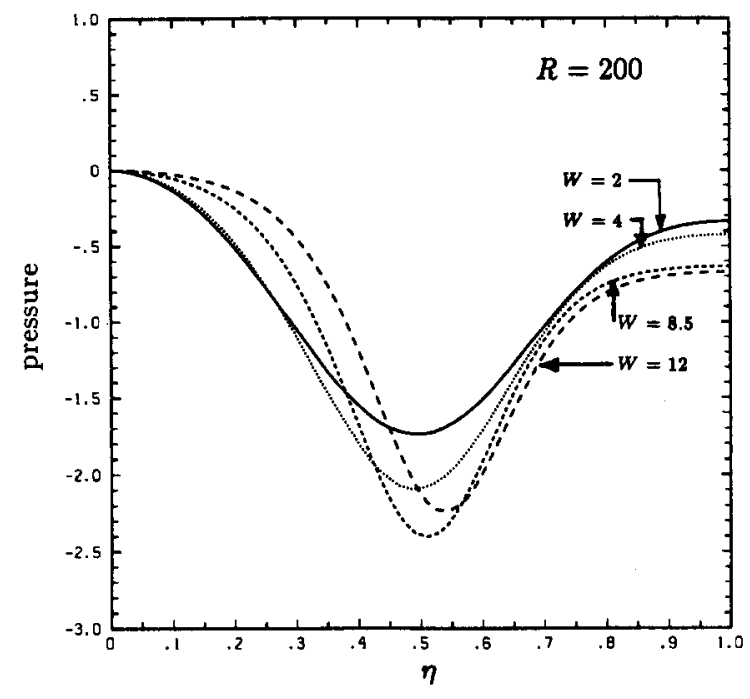

FIG. 7. Pressure distributions along the bubble surface for $R=200$.

biaxial straining flow. Indeed, as this elongation against the flow slows down, the region near the equatorial stagnation plane shows negative curvature $\left(\kappa_{(\eta)}<0\right)$, which increases rapidly as $t$ increases. Therefore, in spite of the fact that the steady bubble shape is identical in the potential flow limit for uniaxial and biaxial straining flows, the transient deformation for supercritical Weber numbers is fundamentally different. A bubble is extended indefinitely in the axial direction in a uniaxial straining flow, while in a biaxial extensional flow the deformation at large times corresponds to an increase of negative curvature $\left(\kappa_{(\eta)}\right)$ along the equatorial plane, without much extension in the axial direction.

The oscillation of a bubble in a biaxial straining flow was also computed for the case $W=1$ in the potential flow limit. Starting with the steady-state solution for $W=2.7$, the Weber number was decreased to 1.0 at $\tilde{t}=0$. In Fig. 9, the radius of the bubble in the axial direction is plotted as a function of a surface tension based time scale $\tilde{t}\left(\tilde{t} \equiv t^{\prime} / \tilde{t}_{c}\right.$, see Sec. II A), and the result is compared with that of a bubble in a uniaxial straining flow. For the uniaxial flow problem, the initial condition is also the steady-state solution for $W=2.7$. As we can see in Fig. 9, the phases are different, but the frequencies are almost the same for the two cases. This result is consistent with the fact that the formula $\omega_{2}^{2}=\omega_{2,0}^{2}$ $\times(1-0.31 W)+o(W)$ is valid for both cases, but extends the result to finite $W$.

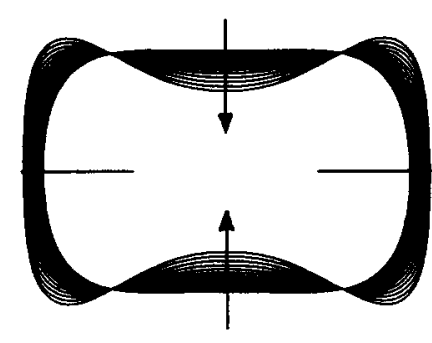

FIG. 8. Consecutive deformations of a bubble in an inviscid, biaxial straining flow at $W=2.9$, starting from the steady state for $W=2.7$ $(1.7<t<4.5)$.

\section{B. Unsteady deformation in high Reynolds number flow}

As shown in Sec. V, a bubble in a biaxial straining flow at a high Reynolds number has a steady shape with negative curvature at the axial stagnation points if the Weber number is sufficiently large (see Fig. 4). In this section, we show that the steady-state shapes obtained in Sec. VI are stable by demonstrating that an initially deformed bubble, with a small disturbance, or even with a large disturbance, is eventually attracted to the steady-state shape obtained via the steadystate analysis.

The first case that we consider is the unsteady deformation of a bubble at $R=100$ and $W=12$, whose initial state is the $R=100$ and $W=10$ steady-state solution. The consecutive bubble shapes are overlapped in Fig. 10. As we can see in Fig. 10, the bubble is attracted directly to the steady-state shape for $R=100$ and $W=12$. In addition, we considered the unsteady deformation of a bubble started from rest for two cases $(R=50 / W=12$ and $R=100 / W=10)$, i.e., at $t=0$, the flow is turned on with the bubble shape initially being spherical. The consecutive bubble shapes are shown in Figs. 11 (a) and 11(b). As we can see, the bubble shapes are smoothly attracted once again to the steady-state shapes. The poles (the intersections of the bubble surface and the symmetry axis) move inward monotonically, except for a nearly negligible overshoot near the steady state. However, the behavior of the surface near the equator is different. Initially, the surface moves inward at the equatorial plane as a result of the high stagnation pressure at the equator. However, very quickly the vorticity levels increase in this region because of the vortex stretching mechanism, the pressure begins to decrease, and the bubble surface subsequently moves monotonically outward. By comparing the $R=50$ / $W=12$ and $R=100 / W=10$ cases, we can see that the initial effect of higher stagnation pressures is more prominent for the higher Reynolds number. However, in both cases, the

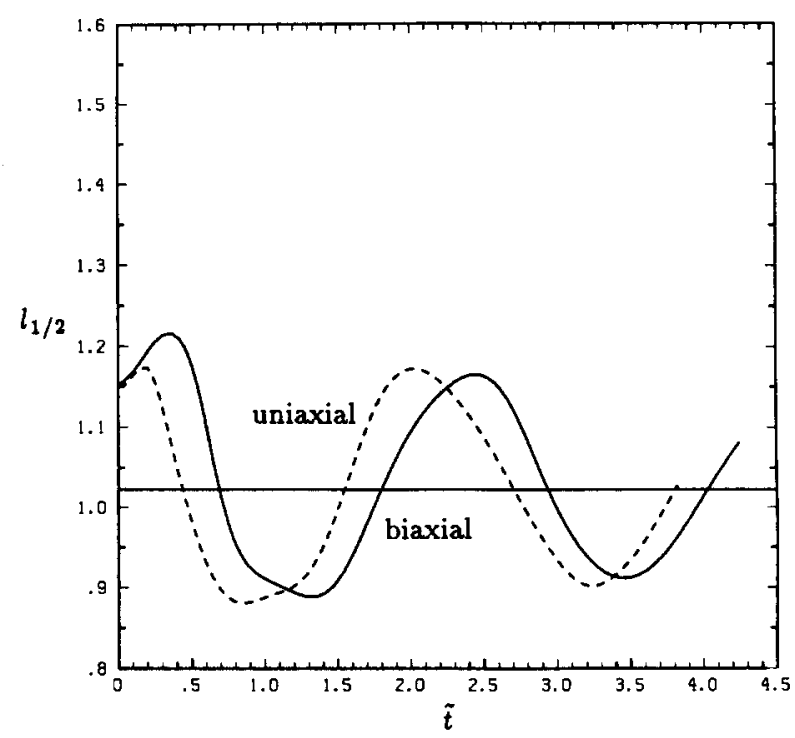

FIG. 9. Oscillation of a bubble in an inviscid, straining flow at $W=1$ (-: biaxial straining flow: - - - uniaxial straining flow; and $\tilde{t}$ is the surface tension based dimensionless time, $\tilde{t}=\sqrt{(2 / W)} t)$. 
FIG. 10. Unsteady deformation of a bubble in a biaxial straining flow at $R=100$ and $W=12$ (starting from the steady solution of $R=100$ / $W=10)$.

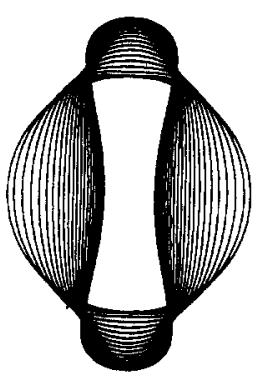

(a)

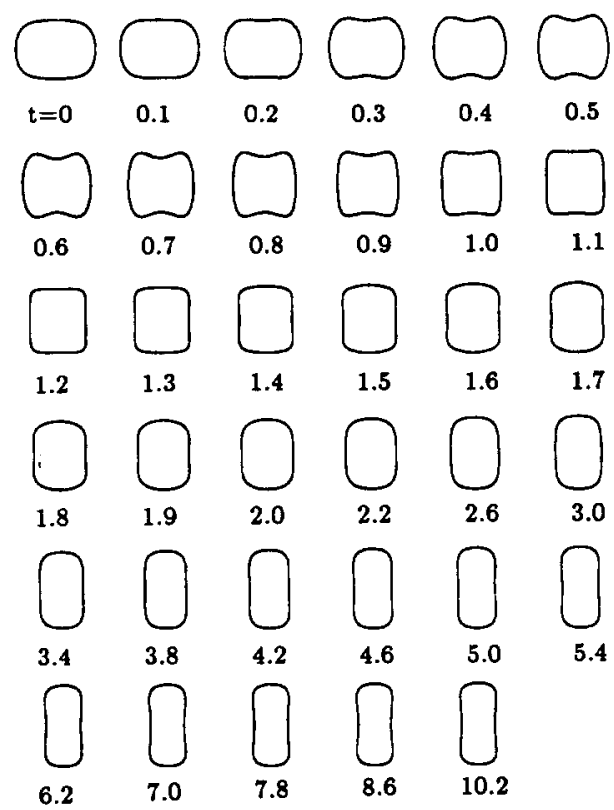

(c)

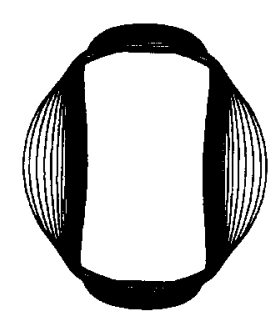

(b)

0.5

1.1

1.7

3.0

5.4

5.4

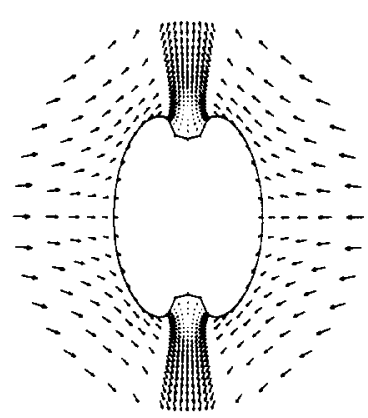

(b)
FIG. 12. (a) Unsteady deformation of a bubble in a biaxial straining flow at $R=100$ and $W=1000$ starting from spherical shape. (b) The velocity field at $t=0.575$.

tions for biaxial straining flows [at least for $R \leqslant 200$ and $W \leqslant O(10)]$ are stable for a very wide range of initial conditions. Formation of the negative curvature $\left(\kappa_{(\eta)}<0\right)$ near the equatorial plane at the early stage of deformation is more prominent than for the case in 11(b). However, later the curvature reverts to positive values as a result of the pressure drop effect discussed previously, and the same steady shape is obtained as before.

Although the decrease in pressure at the equatorial plane because of the increasing vorticity levels seems to provide a self-consistent explanation for the transition in deformation behavior at the equatorial plane, one question that we have not explored directly is the role of surface tension in the transient evolution of the bubble shape. In order to assess the effects of surface tension, we did the same computation for $R=100$ and $W=1000$, where the surface tension effect may be neglected. The consecutive deformations are shown in Fig. 12. As we can see in Fig. 12, the negative curvature grows monotonically and very high curvature edges are formed as time increases. Clearly, surface tension has played a critical role in the previous observations, presumably by limiting the degree of initial deformation at the equatorial plane, so that the vorticity increase/pressure decrease mechanism can come into play [compare Figs. 11(b) and 12(a)]. In this regard, it is interesting to examine the flow field around the deformed bubble at $t=0.575$, as shown in Fig. 12(b). However, it is unclear whether the shape can ever revert to outward movement at the equatorial plane. Finally, it should be mentioned that the present result for $R=100$ / $W=1000$ is not complete, and we do need more systematic analyses for the bubble deformation when the Weber number is asymptotically large.

\section{ACKNOWLEDGMENTS}

This work was supported by grants from the Fluid Mechanics Program and the Office of Advanced Scientific Computing at the National Science Foundation.

'J. O. Hinze, AIChE J. 1, 289 (1955).

${ }^{2}$ D. A. Lewis and J. F. Davidson, Trans. Inst. Chem. Eng. 60, 283 (1982).

${ }^{3}$ I. S. Kang and L. G. Leal, Phys. Fluids 30, 1929 (1987).
FIG. 11. Unsteady deformations of a bubble in a biaxial straining flow: (a) $R=50 / W=12$ starting from the spherical shape, (b) $R=100 / W=10$ starting from the spherical shape, and (c) $R=100 / W=10$ starting from the steady solution of $R=\infty / W=2.7$. 
${ }^{4}$ G. K. Youngren and A. Acrivos, J. Fluid Mech. 76, 433 (1976).

${ }^{5}$ M. J. Miksis, Phys. Fluids 24, 1229 (1981).

${ }^{6}$ G. Ryskin and L. G. Leal, J. Fluid Mech. 148, 37 (1984).

${ }^{7}$ G. I. Taylor, Proc. R. Soc. London Ser. A 146, 501 (1934).

${ }^{8} \mathrm{G}$. I. Taylor, in Proceedings of the 11th International Congress of Applied Mechanics, Munich, 1964, edited by H. Görtler (Springer, Berlin, 1966), p. 790 .

${ }^{9}$ J. D. Buckmaster, J. Fluid Mech. 55, 385 (1972).

${ }^{10}$ D. Barthes-Biesel and A. Acrivos, J. Fluid Mech. 61, 1 (1973).

"A. Acrivos and T. S. Lo, J. Fluid Mech. 86, 641 (1978).
${ }^{12}$ E. J. Hinch, J. Fluid Mech. 101, 545 (1980).

${ }^{13}$ I. S. Kang and L. G. Leal, J. Fuid Mech. 187, 231 (1988).

${ }^{14}$ N. A. Frankel and A. Acrivos, J. Fluid Mech. 44, 65 (1970).

${ }^{15} \mathrm{G}$. K. Batchelor, An Introduction to Fluid Dynamics (Cambridge U.P., Cambridge, 1967).

${ }^{16}$ G. Ryskin and L. G. Leal, J. Fluid Mech. 148, 1 (1984).

${ }^{17}$ G. Ryskin and L. G. Leal, J. Fluid Mech. 148, 19 (1984).

${ }^{18}$ P. J. Roache, Computational Fluid Dynamics (Hermosa, Albuquerque, NM, 1972).

${ }^{19}$ D. Dandy, Ph.D. dissertation, California Institute of Technology, 1987. 